\title{
Application of a Full Reynolds Stress Model to High Lift Flows
}

\author{
E. M. Lee-Rausch, C. L. Rumsey \\ NASA Langley Research Center, Hampton VA 23681-2199, USA \\ B. Eisfeld ${ }^{\ddagger}$ \\ DLR Institute of Aerodynamics and Flow Technology, Braunschweig, D-38108, Germany
}

\begin{abstract}
A recently developed second-moment Reynolds stress model was applied to two challenging high-lift flows: (1) transonic flow over the ONERA M6 wing, and (2) subsonic flow over the DLR-F11 wing-body configuration from the second AIAA High Lift Prediction Workshop. In this study, the Reynolds stress model results were contrasted with those obtained from one- and two-equation turbulence models, and were found to be competitive in terms of the prediction of shock location and separation. For an ONERA M6 case, results from multiple codes, grids, and models were compared, with the Reynolds stress model tending to yield a slightly smaller shock-induced separation bubble near the wing tip than the simpler models, but all models were fairly close to the limited experimental surface pressure data. For a series of high-lift DLR-F11 cases, the range of results was more limited, but there was indication that the Reynolds stress model yielded less-separated results than the one-equation model near maximum lift. These less-separated results were similar to results from the one-equation model with a quadratic constitutive relation. Additional computations need to be performed before a more definitive assessment of the Reynolds stress model can be made.
\end{abstract}

\section{Nomenclature}

$a$

*Research Engineer, Computational AeroSciences Branch, E.Lee-Rausch@nasa.gov. Associate Fellow AIAA.

†Senior Research Scientist, Computational AeroSciences Branch, C.L.Rumsey@nasa.gov. Fellow AIAA.

${ }^{\ddagger}$ Research Scientist, Dept. $C^{2} A^{2} S^{2} E$. Bernhard.Eisfeld@dlr.de, Member AIAA.

wing reference area, $\mathrm{m}^{2}$

wing aspect ratio

wing reference span, $m$

wing reference chord, $\mathrm{m}$

total drag coefficient

total lift coefficient

total pitching moment coefficient

streamwise surface skin friction coefficient, $2 \tau_{w, x} /\left(\bar{\rho}_{\text {inf }} U_{\text {inf }}^{2}\right)$

surface pressure coefficient, $2\left(p-p_{\text {inf }}\right) /\left(\bar{\rho}_{\text {inf }} U_{\text {inf }}^{2}\right)$

Cartesian component of diffusion tensor, $\mathrm{m}^{2} / \mathrm{s}^{3}$

distance to the nearest wall, $m$

Menter's blending function

fully turbulent

alternate scale-determining variable, $\sqrt{1 / \omega}, \mathrm{s}^{1 / 2}$

specific kinetic turbulence energy, $\mathrm{m}^{2} / \mathrm{s}^{2}$

freestream Mach number

normalized rotation tensor used in quadratic constitutive relation correction

two-equation model production term, $\tau_{i j}\left(\partial \hat{u}_{i} / \partial x_{j}\right), \mathrm{kg} /\left(\mathrm{ms}^{3}\right)$

Cartesian component of Reynolds-stress production tensor, $\mathrm{m}^{2} / \mathrm{s}^{3}$ 


\begin{tabular}{|c|c|}
\hline$p$ & pressure, $\mathrm{kg} /\left(\mathrm{ms}^{2}\right)$ \\
\hline$\hat{R}_{i j}$ & Cartesian component of specific Reynolds-stress tensor, $\mathrm{m}^{2} / \mathrm{s}^{2}$ \\
\hline$R e_{c}$ & freestream Reynolds number based on $c_{r e f}$ length scale, $U_{i n f} c_{r e f} / \bar{\nu}_{i n f}$ \\
\hline$\hat{S}$ & modified vorticity production term in Spalart-Allmaras model, 1/s \\
\hline$t$ & time, $\mathrm{s}$ \\
\hline$\hat{u}_{i}$ & Cartesian component of velocity vector, $\mathrm{m} / \mathrm{s}$ \\
\hline$\overline{u^{\prime}}, \overline{v^{\prime}}, \overline{w^{\prime}}$ & turbulent velocity fluctuations, $\mathrm{m} / \mathrm{s}$ \\
\hline$U$ & total velocity magnitude, $\mathrm{m} / \mathrm{s}$ \\
\hline$W_{i j}$ & Cartesian component of vorticity tensor, $1 / \mathrm{s}$ \\
\hline$x_{i}$ & Cartesian coordinate, $\mathrm{m}$ \\
\hline$\alpha$ & angle of attack, deg. \\
\hline$\varepsilon_{i j}$ & Cartesian component of dissipation-rate tensor, $\mathrm{m}^{2} / \mathrm{s}^{3}$ \\
\hline $\bar{\mu}$ & molecular (dynamic) viscosity, $\mathrm{kg} /(\mathrm{ms})$ \\
\hline $\bar{\nu}$ & kinematic viscosity, $\mathrm{m}^{2} / \mathrm{s}$ \\
\hline$\hat{\nu}$ & Spalart-Allmaras turbulent working variable, $\mathrm{m}^{2} / \mathrm{s}$ \\
\hline$\Pi_{i j}$ & Cartesian component of pressure-strain tensor, $\mathrm{m}^{2} / \mathrm{s}^{3}$ \\
\hline $\bar{\rho}$ & density, $\mathrm{kg} / \mathrm{m}^{3}$ \\
\hline$\tau_{w}$ & wall shear stress, $\mathrm{kg} /\left(\mathrm{ms}^{2}\right)$ \\
\hline$\tau_{i j}$ & turbulent stress tensor, $\mathrm{kg} /\left(\mathrm{ms}^{2}\right)$ \\
\hline$\Omega$ & magnitude of vorticity, $\sqrt{2 W_{i j} W_{i j}}, 1 / \mathrm{s}$ \\
\hline$\omega$ & specific dissipation rate, $1 / \mathrm{s}$ \\
\hline Subsc & \\
\hline $\inf$ & freestream conditions \\
\hline$t$ & turbulent \\
\hline
\end{tabular}

\section{Introduction}

Second-moment differential Reynolds stress models (RSMs) are sometimes considered too difficult to use for complex aerodynamic flows. Also, although they involve solving seven equations for the turbulence model, to date they have not consistently proved to be worth the additional effort or expense compared with simpler one- or two-equation models. The second-moment differential Reynolds stress model SSG/LRR-RSM-w2012, developed by Eisfeld et al. ${ }^{1}$ at DLR, was recently implemented in the NASA codes CFL3D and FUN3D. An extensive effort was undertaken by Eisfeld et al. ${ }^{2}$ to verify the implementations in comparison with results from the DLR code TAU. Many verification and validation results from this turbulence model have also been included on the NASA Turbulence Modeling Resource (TMR) website (http://turbmodels.larc.nasa.gov).

The SSG/LRR-RSM-w2012 is an omega-based model that uses a blended form of the pressure-strain model, with Menter's " $F_{1}$ " function ${ }^{3}$ used for the blending. Rumsey ${ }^{4}$ showed that this model performs better in general than the omega-based Wilcox stress-omega Reynolds stress model, ${ }^{5}$ which relies solely on the Launder-Reece-Rodi (LRR $)^{6}$ pressure strain component. The Wilcox stress-omega model has been investigated by Rodio et al. ${ }^{7}$ and Thompson and Hassan ${ }^{8}$ for a variety of cases, including high lift flows. Results were shown in the case of the NASA Trapezoidal wing-body to be similar in character to SST model $^{3}$ results, with both tending to underpredict the lift near $C_{L, \max }$. The Spalart-Allmaras (SA) model ${ }^{9}$ has generally performed much better for this particular case at such conditions. For the DLR-F11 wing-body studied in the second AIAA High Lift Prediction Workshop (HiLiftPW-2), Thompson and Hassan reported that the Wilcox stress-omega model performed similarly to SA on the particular grid (with no brackets) employed by the authors; neither result agreed well with experiment near $C_{L, \max }$.

In the current study, the second-moment Reynolds stress model SSG/LRR-RSM-w2012 is applied to two challenging high-lift flows: (1) transonic flow over the ONERA M6 wing, and (2) subsonic flow over the DLR-F11 wing-body configuration from the second AIAA High Lift Prediction Workshop. For the first case, an extensive investigation of the ONERA M6 wing configuration is performed, including grid, code, and turbulence model effects. For the second DLR F11 case, we compare the performance of the SSG/LRR-RSM-w2012-SD model with various forms of the SA one-equation model for high lift flow cases from the HiLiftPW-2 workshop, ${ }^{10}$ building upon prior FUN3D ${ }^{11}$ analysis of the HiLiftPW-2 test cases. The workshop configuration, the DLR-F11 wing-body, is a three-element wing in landing configuration. There 
are three cases associated with the workshop, with increasing geometric fidelity. The first case includes no support brackets, the second includes support brackets, and the third includes both support brackets as well as idealized representations of pressure tube bundles, which were present in the experimental campaign. In the summary of workshop results, ${ }^{10}$ the support brackets were found to be influential in causing large wedge-shaped regions of separated flow on the main element near stall. The pressure tube bundles included alongside the slat brackets also had an influence on the computed flowfields near maximum lift. Grid refinement studies for the DLR-F11 configuration were not performed in this study. However, in an attempt to provide some assessment of the influence of grid, a limited number of FUN3D computations were made on different grid systems from the workshop.

\section{Description of Turbulence Models}

The primary focus of this paper is the SSG/LRR-RSM-w2012 second-moment Reynolds stress model. However, because its results are compared to other (simpler) models, very brief descriptions of those are also provided.

\section{II.A. SSG/LRR-RSM-w2012 Model and Variants}

Documentation of these models can be found in Eisfeld et al. ${ }^{12}$ as well as on the NASA TMR website (http://turbmodels.larc.nasa.gov). The six Reynolds stress transport equations and one length scale transport equation are given by:

$$
\begin{gathered}
\frac{\partial \bar{\rho} \hat{R}_{i j}}{\partial t}+\frac{\partial\left(\bar{\rho} \hat{u}_{k} \hat{R}_{i j}\right)}{\partial x_{k}}=\bar{\rho} P_{i j}+\bar{\rho} \Pi_{i j}-\bar{\rho} \varepsilon_{i j}+\bar{\rho} D_{i j} \\
\frac{\partial(\bar{\rho} \omega)}{\partial t}+\frac{\partial\left(\bar{\rho} \hat{u}_{k} \omega\right)}{\partial x_{k}}=\frac{\alpha_{\omega} \omega}{\hat{k}} \frac{\bar{\rho} P_{k k}}{2}-\beta_{\omega} \bar{\rho} \omega^{2}+\frac{\partial}{\partial x_{k}}\left[\left(\bar{\mu}+\sigma_{\omega} \frac{\bar{\rho} \hat{k}}{\omega}\right) \frac{\partial \omega}{\partial x_{k}}\right]+\sigma_{d} \frac{\bar{\rho}}{\omega} \max \left(\frac{\partial \hat{k}}{\partial x_{j}} \frac{\partial \omega}{\partial x_{j}}, 0\right)
\end{gathered}
$$

where $\bar{\rho} \hat{R}_{i j}=-\tau_{i j}$ is the Reynolds stress tensor, $\hat{u}_{k}$ is the mean flow velocity vector, $P_{i j}$ represents production, $\Pi_{i j}$ represents pressure strain, $\varepsilon_{i j}$ represents the dissipation, and $D_{i j}$ represents the diffusion. Furthermore, $\omega$ is the specific dissipation rate, $\hat{k}$ is the turbulent kinetic energy, $\bar{\mu}$ is the molecular dynamic viscosity, $\bar{\rho}$ is the mean flow density, and $\alpha_{\omega}, \beta_{\omega}, \sigma_{\omega}$, and $\sigma_{d}$ are constants. The pressure-strain coefficients are blended between $\mathrm{LRR}^{6}$ near walls (without wall-correction terms) and Speziale-Sarkar-Gatski (SSG) ${ }^{13}$ away from walls.

The SSG/LRR-RSM-w2012 model makes use of the Daly-Harlow gradient diffusion model. ${ }^{14}$ However, for some complex geometry applications, this version has proved to be less robust than the SSG/LRR-RSMw2012-SD version that uses a "simplified diffusion" model. ${ }^{15}$ In Rumsey ${ }^{4}$ as well as on the TMR website, results from both versions of the model have been shown to be very similar for a variety of test cases. The simplified SD version has been used for all DLR-F11 results shown here. Additional details for this model are not given here, but can be found in the original reference. ${ }^{12}$

A relatively new variant of this model, described in Togiti and Eisfeld, ${ }^{16}$ solves for $g(\equiv \sqrt{1 / \omega})$ instead of $\omega$. The equation is derived by recasting Eq. (2) in terms of $g$. However, to avoid $g$ reaching very small values near boundary layer edges, a modification to one of the terms in the $g$-equation is required, as detailed in Ref. 12. This RSM-g variant has been shown to yield similar predictions as the SSG/LRR-RSM-w2012 model, while demonstrating less dependency on near-wall grid resolution (because of its simpler boundary condition of $g=0$ at solid walls). Note that the second-moment Reynolds stress models such as those described here should inherently account for curvature effects as well as normal stress anisotropy unlike the one- and two-equation models that require explicit corrections.

\section{II.B. SA Model and Variants}

The Spalart-Allmaras one-equation model $^{9}$ is very widely used for aerodynamic applications. The equation for the turbulence working variable $(\hat{\nu})$ is: 


$$
\frac{\partial \hat{\nu}}{\partial t}+\hat{u}_{j} \frac{\partial \hat{\nu}}{\partial x_{j}}=c_{b 1}\left(1-f_{t 2}\right) \hat{S} \hat{\nu}-\left[c_{w 1} f_{w}-\frac{c_{b 1}}{\kappa^{2}} f_{t 2}\right]\left(\frac{\hat{\nu}}{d}\right)^{2}+\frac{1}{\sigma}\left[\frac{\partial}{\partial x_{j}}\left((\bar{\nu}+\hat{\nu}) \frac{\partial \hat{\nu}}{\partial x_{j}}\right)+c_{b 2} \frac{\partial \hat{\nu}}{\partial x_{i}} \frac{\partial \hat{\nu}}{\partial x_{i}}\right]
$$

where $\hat{S}$ is a modified vorticity production term, $d$ is distance to the wall, $\bar{\nu}$ is molecular kinematic viscosity, and the resulting eddy viscosity is a function of $\hat{\nu}$. Other terms are functions and constants, not described here, but can be found in the original reference or on the NASA TMR website (http://turbmodels.larc.nasa.gov).

In the rotation/curvature (RC) correction of Shur et al., ${ }^{17}$ the $c_{b 1} \hat{S} \hat{\nu}$ term gets multiplied by a rotation term that is a function of system rotation rate (for moving reference frames), the vorticity tensor, and the strain rate tensor and its material derivative. This correction is designed to account for streamline curvature effects (which, for example, are known to reduce eddy viscosity near convex walls and enhance it near concave walls).

In the quadratic constitutive relation (QCR) correction of Spalart ${ }^{18}$ — which can be applied to any linear eddy viscosity model - the turbulence transport equation itself is not altered at all, but the following form is used for the turbulent stresses:

$$
\tau_{i j, Q C R}=\tau_{i j}-C_{c r 1}\left[O_{i k} \tau_{j k}+O_{j k} \tau_{i k}\right]
$$

where $C_{c r 1}=0.3, \tau_{i j}$ are the turbulent stresses computed from the linear Boussinesq relation, and $O_{i k}$ is an antisymmetric normalized rotation tensor, defined by:

$$
O_{i k}=2 W_{i k} / \sqrt{\frac{\partial u_{m}}{\partial x_{n}} \frac{\partial u_{m}}{\partial x_{n}}}
$$

This QCR relation is designed to approximately account for the normal stress anisotropy typically present in simple boundary layer flows $\left(\overline{u^{\prime 2}}>\overline{w^{\prime 2}}>\overline{v^{\prime 2}}\right)$, but it has proved to be useful in complex flows as well. When QCR is used in combination with the SA model with RC correction, it is referred to as SA-RC-QCR2000.

\section{II.C. SST and SST-V Models}

Like the one-equation SA model, the two-equation SST model ${ }^{3}$ is a widely-used linear eddy-viscosity (Boussinesq) model. It solves the two equations:

$$
\begin{gathered}
\frac{\partial(\bar{\rho} \hat{k})}{\partial t}+\frac{\partial\left(\bar{\rho} \hat{u}_{j} \hat{k}\right)}{\partial x_{j}}=P-\beta^{*} \bar{\rho} \omega \hat{k}+\frac{\partial}{\partial x_{j}}\left[\left(\bar{\mu}+\sigma_{k} \mu_{t}\right) \frac{\partial \hat{k}}{\partial x_{j}}\right] \\
\frac{\partial(\bar{\rho} \omega)}{\partial t}+\frac{\partial\left(\bar{\rho} \hat{u}_{j} \omega\right)}{\partial x_{j}}=\frac{\gamma}{\bar{\nu}_{t}} P-\beta \bar{\rho} \omega^{2}+\frac{\partial}{\partial x_{j}}\left[\left(\bar{\mu}+\sigma_{\omega} \mu_{t}\right) \frac{\partial \omega}{\partial x_{j}}\right]+2\left(1-F_{1}\right) \frac{\bar{\rho} \sigma_{\omega 2}}{\omega} \frac{\partial \hat{k}}{\partial x_{j}} \frac{\partial \omega}{\partial x_{j}}
\end{gathered}
$$

The SST-V variant is identical to SST, except that it uses an approximation for the production term $P$, basing it on vorticity instead of strain rate. The eddy viscosity in SST and SST-V is a function of $\bar{\rho}, \hat{k}, \omega$, and local magnitude of vorticity, $\Omega$.

Additional details about these models are not provided here, but can be found in the original reference or on the NASA TMR website (http://turbmodels.larc.nasa.gov). There are various curvature correction fixes in the literature for the SST model, but none are used in this study, so they will not be described. Spalart's QCR correction can also be used in conjunction with SST or SST-V, but this was not done in the current study.

\section{Numerical Methods}

Three different flow solvers used in this study are described below. In all codes, the turbulence model equations were solved with first-order spatial accuracy for the advection terms. 


\section{III.A. CFL3D Flow Solver}

CFL3D $^{19}$ is a structured-grid upwind multi-zone CFD code that solves the generalized thin-layer or full Navier-Stokes equations. In the current study, the full viscous terms are used for all computations. CFL3D can use point-matched, patched, or overset grids and employs local time-step scaling, grid sequencing and multigrid to accelerate convergence to steady state. CFL3D is a cell-centered finite-volume method. It uses third-order upwind-biased spatial differencing on the convective and pressure terms, and second-order differencing on the viscous terms; it is globally second-order accurate. Roe's flux difference-splitting method ${ }^{20}$ is used to obtain fluxes at the cell faces. The solution is advanced in time with an implicit approximate factorization method. For each loosely coupled iteration, the mean flow equations are advanced in time with the eddy-viscosity fixed; then the turbulence model is advanced in time with the mean flow solution fixed.

\section{III.B. FUN3D Flow Solver}

FUN3D ${ }^{21-23}$ is a finite-volume Navier-Stokes solver in which the flow variables are stored at the nodes of the mesh. FUN3D solves the equations on mixed element grids, including tetrahedra, pyramids, prisms and hexahedra. At interfaces between neighboring control volumes, the inviscid fluxes are computed with an approximate Riemann solver based on the values on either side of the interface. Several convective flux schemes are available in FUN3D. The most common scheme for subsonic and transonic flows is Roe's flux difference splitting, ${ }^{20}$ which is used in the current study. For second-order accuracy, interface values are obtained by extrapolation of the control volume centroidal values with unweighted least-squares gradients computed at the mesh vertices. Several reconstruction limiters are available in FUN3D. The Venkatakrishnan limiter $^{24}$ is used in the current study for the ONERA M6 cases. For non-tetrahedral meshes, a GreenGauss approach to computing velocity gradients can lead to odd-even decoupling, so edge-based gradients are combined with Green-Gauss gradients, which improves the h-ellipticity of the operator and allows the complete viscous stresses to be evaluated. ${ }^{25}$ The solution at each time-step is updated with a backwards Euler time-integration scheme. At each time step, the linear system of equations is approximately solved with either a multi-color point-implicit procedure. ${ }^{26}$ Local time-step scaling is employed to accelerate convergence to steady state. Similar to CFL3D, the turbulence model equations and the mean flow equations are solved in a loosely-coupled fashion.

\section{III.C. TAU Flow Solver}

The TAU code $^{27}$ solves the RANS equations on mixed-element, unstructured grids with second-order spatial accuracy. It is a node-centered code. Standard numerical methods include a central discretization of the convective RANS terms with artificial dissipation, whereas first- or second-order upwinding is used for the turbulence equations. Alternatively, low dissipation second-order upwind schemes like AUSMDV can be used for the whole set of equations. Integration is carried out either by an explicit three-stage Runge-Kutta or an implicit LUSGS scheme, both accelerated by multigrid. In the current study, a second-order central discretization with matrix-dissipation scheme is used for the mean-flow equations coupled to a first-order upwind scheme for the turbulence equations, and time-integration is carried out with a LUSGS scheme. For the RSM, convergence is accelerated with a three-level multigrid algorithm for the mean flow equations only. For the one-equation model (SA-neg), convergence is accelerated by a 4-level multigrid algorithm for all equations. Local time-step scaling is employed to accelerate convergence to steady state except as noted in the results section. Unlike CFL3D and FUN3D, the turbulence model equations and the mean flow equations are solved in a closely-coupled fashion.

\section{ONERA M6 Results}

In spite of uncertainties associated with the effects of a wing-root splitter plate in the experiment, the ONERA M6 wing ${ }^{28}$ is well known and is often used in transonic-flow CFD validation studies. ${ }^{29,30}$ The M6 wing is challenging not only for the highly three-dimensional nature of its flow, but also for its upper surface shock and shock-induced flow separation near the wing tip. These features are quite prominent even at $3^{\circ}$ and $4^{\circ}$ angle of attack. The M6 wing was run at a Reynolds number of $11.81 \times 10^{6}$ based on mean aerodynamic chord, freestream Mach number of 0.84 , a freestream temperature of $540^{\circ}$ Rankine, and at $4.08^{\circ}$ angle of attack. 
To date, the case has been run on several different grids. The baseline structured grid has a C-O-O topology, with approximately 24 million cells $(865 \times 193 \times 145)$ and 99 thousand surface cells. The baseline unstructured grid, generated with the VGRID v4.0 advancing-layer and advancing-front grid generation software package, ${ }^{31}$ has 6.6 million grid nodes, with approximately 107 thousand wing surface points. Two other grids were obtained from DLR. These are named: "DLR Grid 1" (with 4.5 million grid points and 90 thousand surface points) and "DLR BTE-2" (with 14 million grid points and 80 thousand surface points). The "DLR BTE-2" mesh is a structured mesh that has been converted to an unstructured hexahedral mesh. The baseline unstructured grid and the "DLR Grid 1" grid have a combination of prisms in the near-wall region and tetrahedra in the rest of domain. For all of these grids, the grid normal spacing is set such that an average normalized coordinate $\mathrm{y}^{+}$is less than one for the first grid cell at the wall for the majority of the wing. A view of the upper-surface grid distributions from all four grids is given in Fig. 1.

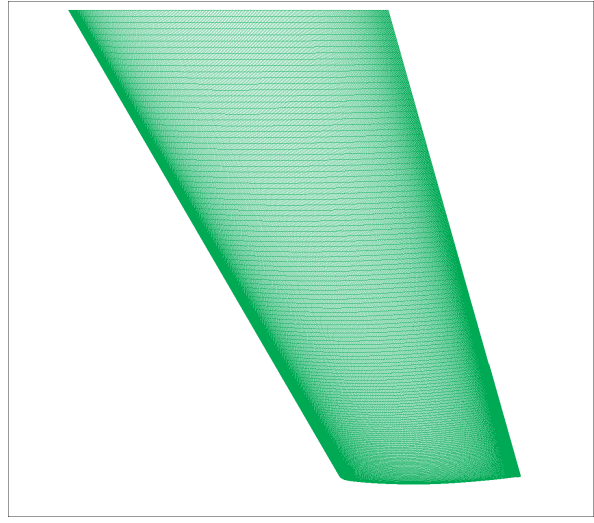

(a) Fine structured baseline grid

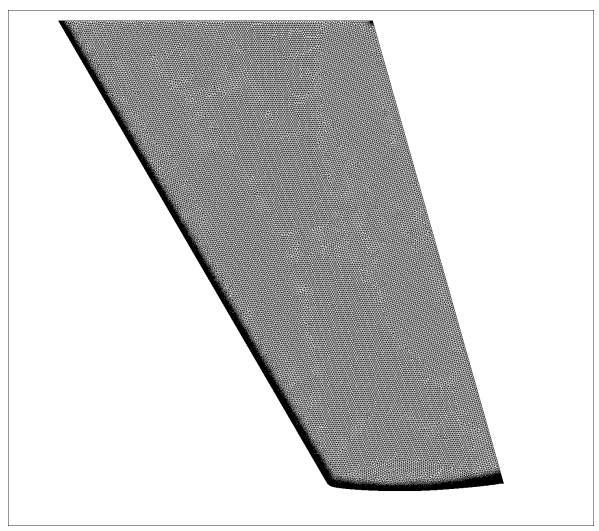

(c) Fine unstructured "DLR Grid 1"

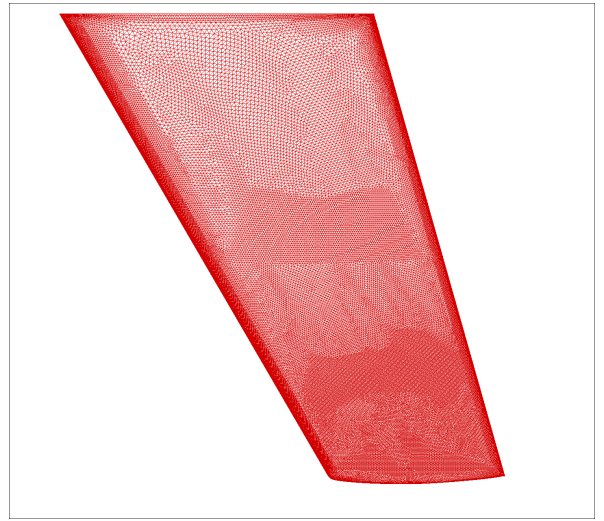

(b) Fine unstructured baseline grid

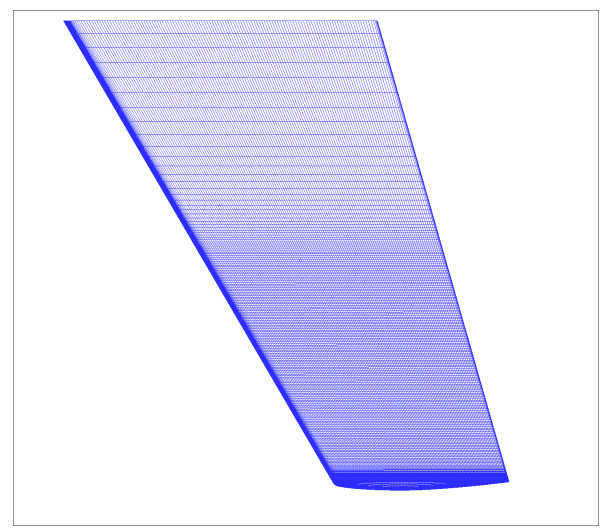

(d) Fine structured "DLR BTE-2"

Figure 1. ONERA M6 computational surface grids.

A summary of pressure coefficient results is shown in Fig. 2 at several outboard span stations on the wing from all the codes, models and grids. Results from the SSG/LRR-RSM-w2012 model (referred to in the figure simply as "RSM") are also compared to SA and SST-V results for both CFL3D and FUN3D for the fine baseline structured and unstructured grids. Results from the TAU code with the SA-neg and RSM-g variants are also included for the "DLR BTE-2" and "DLR Grid 1" grids as well as additional FUN3D results for the "DLR Grid 1" grid. (Note that the TAU computations with SSG/LRR-RSM-w2012 model on grid BTE-2 have been carried out in time-accurate mode using dual-time stepping, whereas all other computations were carried out, assuming a steady state right from the beginning.) At the $\eta=0.65$ and $\eta=0.80$ span stations, the results show small differences from each other primarily in the prediction of the shock location, with the shock at these stations predicted downstream from the experiment for all models. Similarly, near the tip at $\eta=0.96$ span station, the predicted results vary primarily in the region of the shock. However, at the $\eta=0.90$ span station, the results show a larger variation in the prediction of the shock location and also show larger variations in the separation region downstream of the shock. The band 
of predicted shock locations surrounds the experimental data.

Results at $\eta=0.80$ are further analyzed in Fig. 3. For clarity, the summary plot for all the cases is repeated in Fig. 3(a), then the results from the one- and two-equation models are individually summarized in Fig. 3(b) and the results from the RSM models are summarized in Fig. 3(c). The variation between all codes, models and grids shown in Fig. 3(a) is similar to the variation between the one- and two-equation models shown in Fig. 3(b) and the variation between the RSM models shown in Fig. 3(c). The TAU results on "DLR Grid 1" and "DLR BTE-2" for the RSM as well as the SA-neg model show very small differences at this span location (curves overlay) and predict the shock location slightly further upstream from the FUN3D RSM model computations from "DLR Grid 1". A summary of the FUN3D results for the fine unstructured grid are shown in Fig. 3(d) for all the models, including additionally SA-RC-QCR2000. The shock location from RSM is somewhat downstream from the other models, and the SA and SA-RC-QCR2000 results differ only slightly. For the TAU results, the differences in the SA-neg and the RSM are significantly smaller.

A similar set of plots is shown in Fig. 4 for $\eta=0.90$. At this span station, the variation between all codes, models and grids shown in Fig. 4(a) is much larger than the variation between the one- and two-equation models shown in Fig. 4(b) and the variation between the RSM models shown in Fig. 4(c). As a group, for the one- and two-equation models the shock is predicted farther upstream than for the RSM, and there is a larger region of shock-induced separation.

Comparisons of surface-restricted streamlines shown in Fig. 5 for a subset of computations illustrate the differences in the flow feature predictions on the outboard region of the wing. In Figs. 5(a)-(d), the RSM computations predict smaller areas of shock-induced separation than the SA models for Grid 1. This was characteristic of all the codes and grids in this study. For this case, the RSM has predicted a shorter separation bubble than the other models, and the shock locations for the RSM solutions are downstream of the experimental data. Although not shown here, the one- and two-equation models tend to predict more negative streamwise skin-friction values than RSM in the separated region behind the shock. This is similar to the trend shown in Ref. 12 for the transonic bump test case, where the one- and two-equation models predicted more negative streamwise skin-friction values in the separated region behind the shock.

The flow patterns on the inboard part of the wing were very similar for all cases with one exception, as illustrated in Fig. 5(e). The FUN3D results for SA (and SST-V, not shown) on the fine unstructured grid show an area of spanwise flow just downstream of the shock over the mid-span of the wing. This flow pattern is not seen for the FUN3D RSM result for the same grid (Fig. 5(f)) or for the FUN3D SA results on the DLR Grid 1. Note that although DLR Grid 1 has less nodes than the baseline unstructured grid, the DLR Grid 1 has a finer spatial resolution on the inboard portion of the upper-wing surface. This suggests that insufficient upper surface grid resolution in the unstructured baseline grid is responsible for the additional tiny strip of separation seen in Fig. 5(e). Also, this flow pattern does not seem to significantly impact the predicted pressure at $\eta=0.65$ as shown in Fig. 2 (a).

In Ref. 32, the SA and SST computations for this case exhibited a significant grid-dependent overprediction of separation at $\eta=0.80$. However, in this study the SA and SST computations were consistent across multiple codes, models and grids. While the SA and SST-V models predicted more shock-induced separation than the RSM, these models did not exhibit a significant deviation from the experimental data. Thus, all models appear to be competitive for this test case and set of grids; the experimental data is not detailed enough to definitively discriminate between them.

\section{DLR-F11 Results}

\section{V.A. Workshop Configuration}

The HiLiftPW-2 test cases were based on the European High Lift Programme (EUROLIFT) DLR-F11 high lift landing configuration, which was tested in the Low Speed Wind Tunnel of Airbus in Bremen (B-LSWT) and the European Transonic Wind Tunnel (ETW) in Cologne. ${ }^{33}$ The configuration is representative of a commercial wide-body twin-jet high-lift configuration with three continuous elements: full-span slat, main and full-span flap. The model was tested at low Reynolds number $\left(R e_{c}=1.35 \times 10^{6}\right)$ in the B-LSWT as well as high Reynolds number $\left(R e_{c}=15 \times 10^{6}\right)$ in the ETW. A significant amount of high-quality surface and flowfield data are available, including data for an assessment of Reynolds number scale effects. Forces, moments and surface pressures were measured in both facilities. The total force and moment measurements include the body (fuselage) contributions. Although multiple slat and flap settings were tested, the workshop test cases focused on a landing configuration $\left(26.5^{\circ}\right.$ slat angle and $32^{\circ}$ flap angle). The reference geometric 


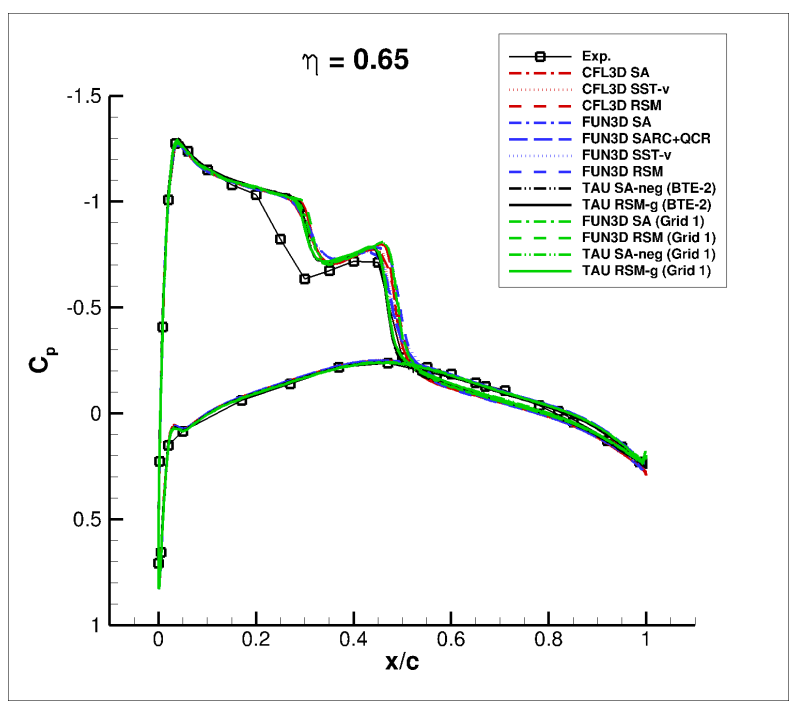

(a) $65 \%$ span station

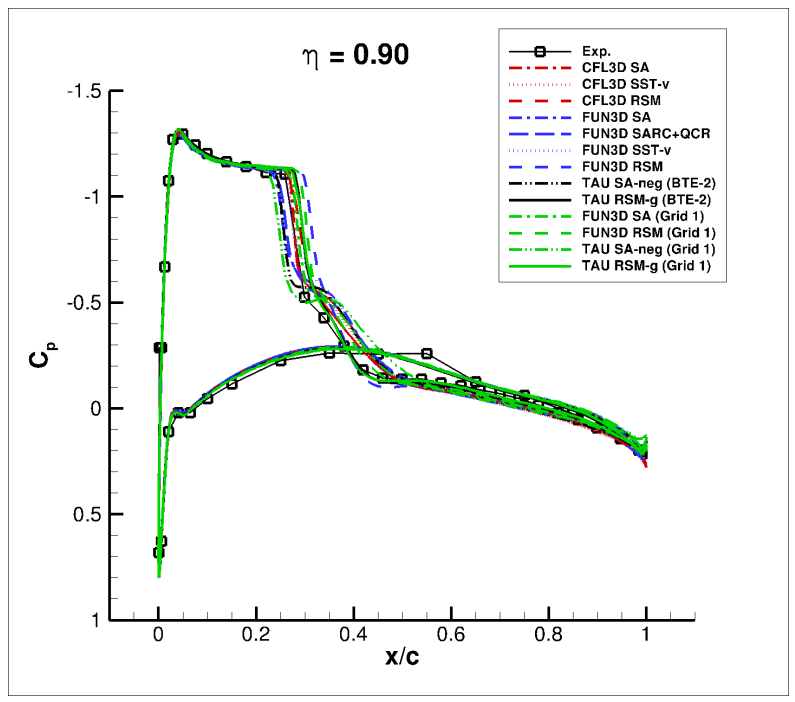

(c) $90 \%$ span station

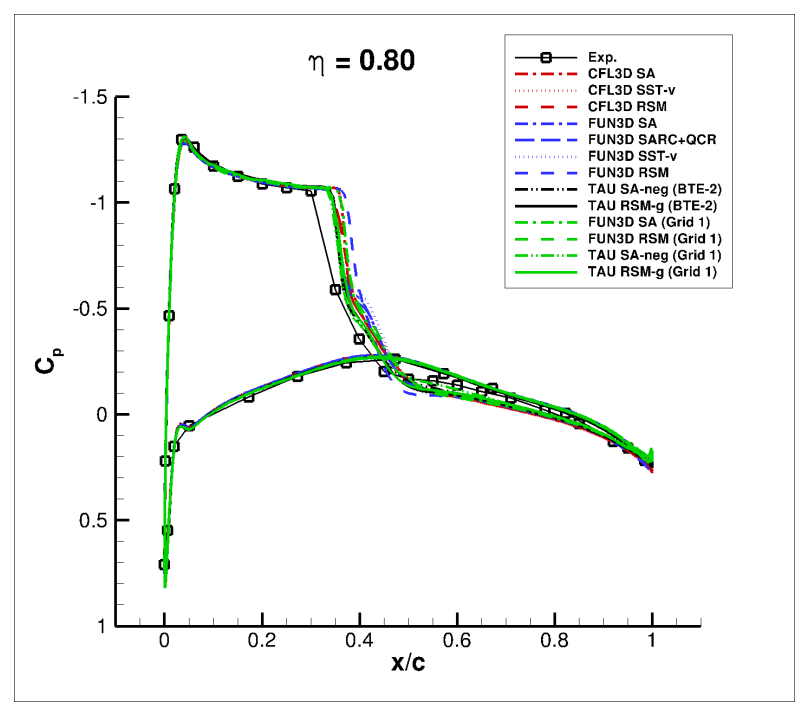

(b) $80 \%$ span station

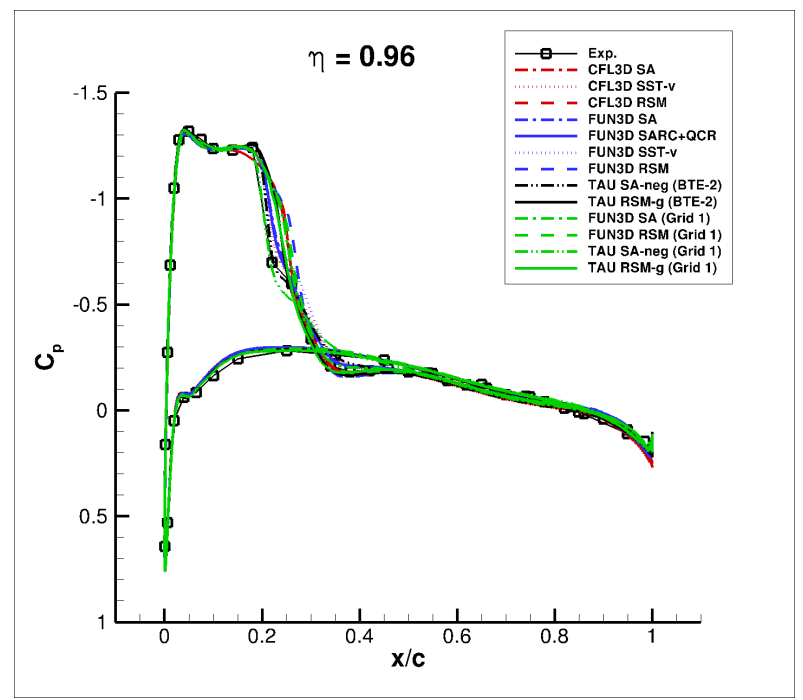

(d) $96 \%$ span station

Figure 2. Comparison of ONERA M6 sectional pressure coefficient from all codes, models and grids. 


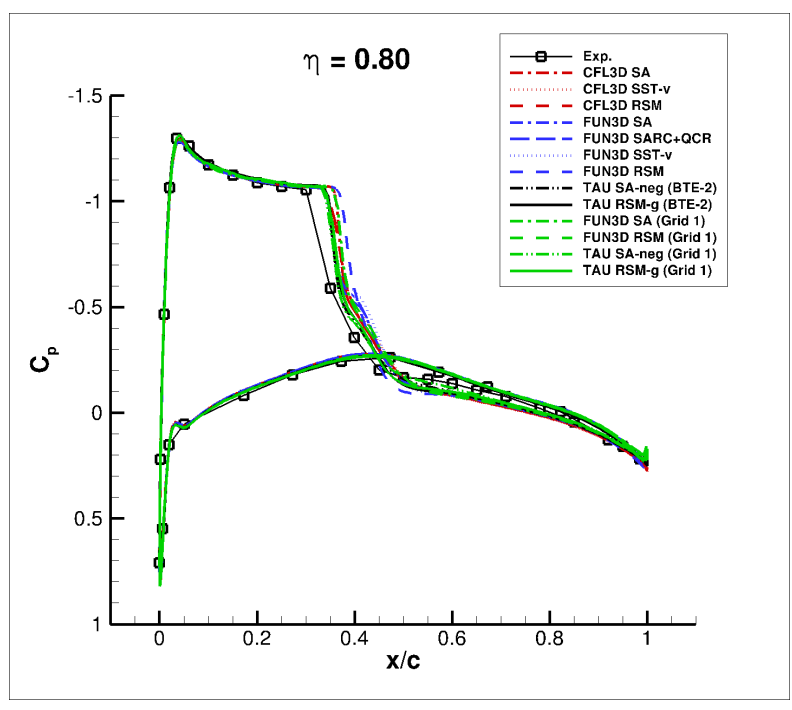

(a) All codes, models and grids

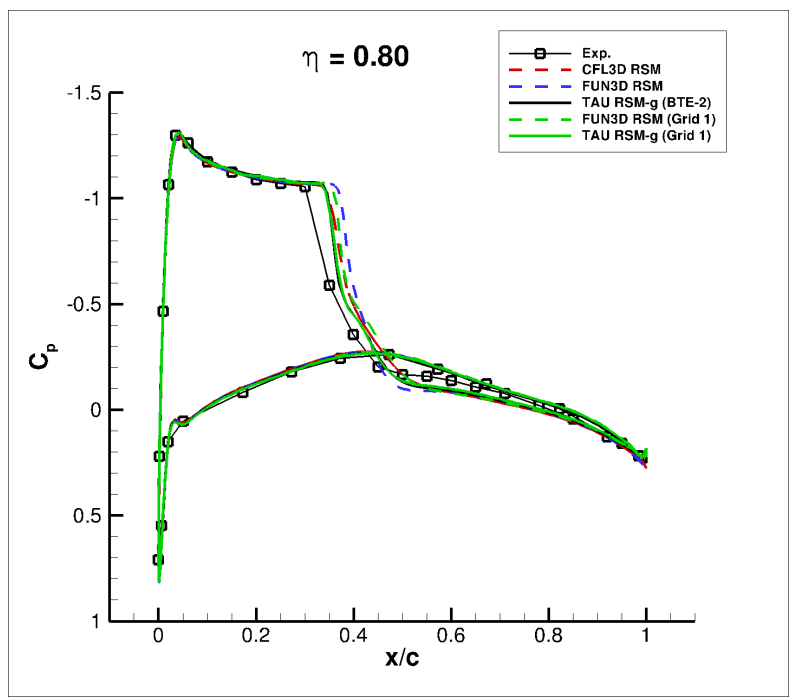

(c) RSM models

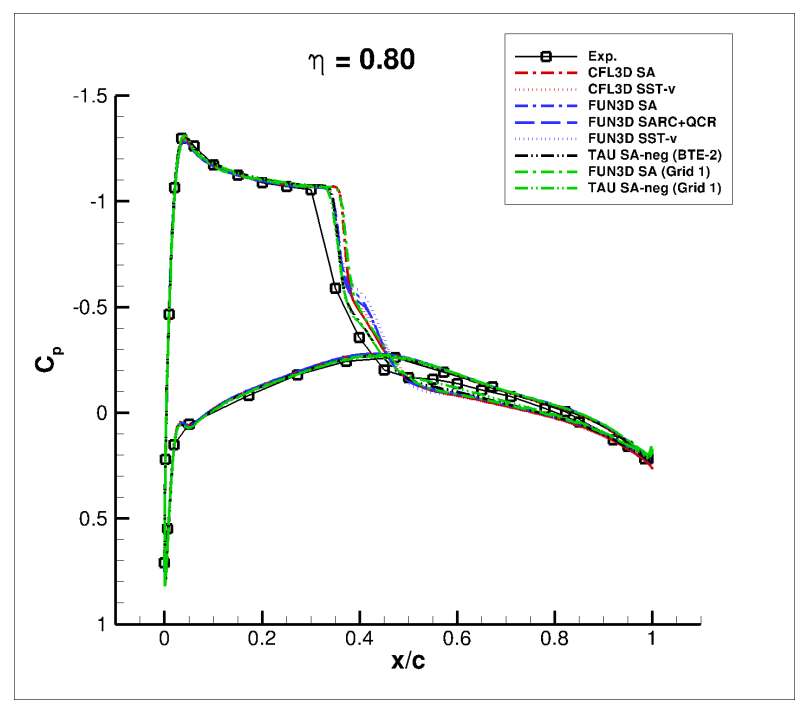

(b) One and two equation models

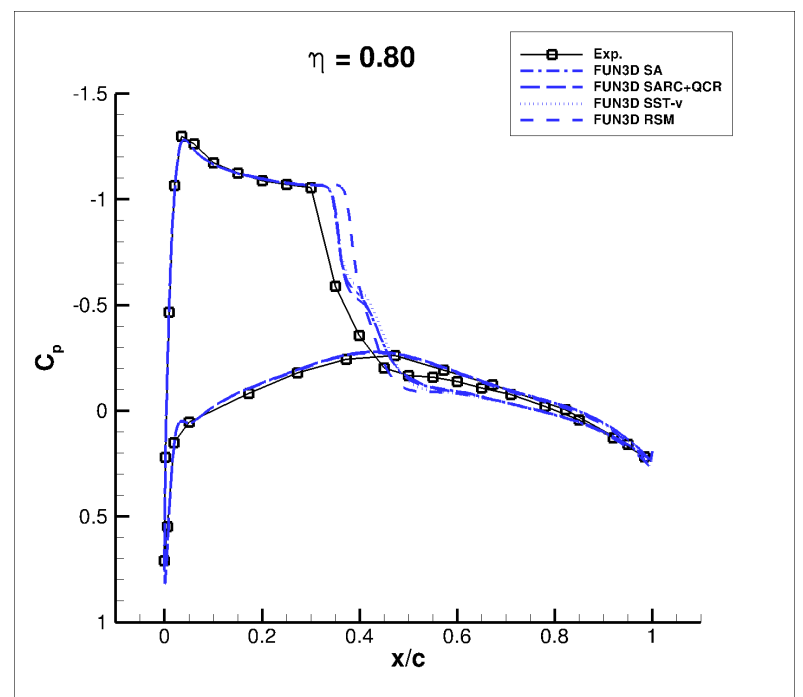

(d) All models, one code and one grid

Figure 3. Comparison of ONERA M6 sectional pressure coefficient at $80 \%$ span station. 


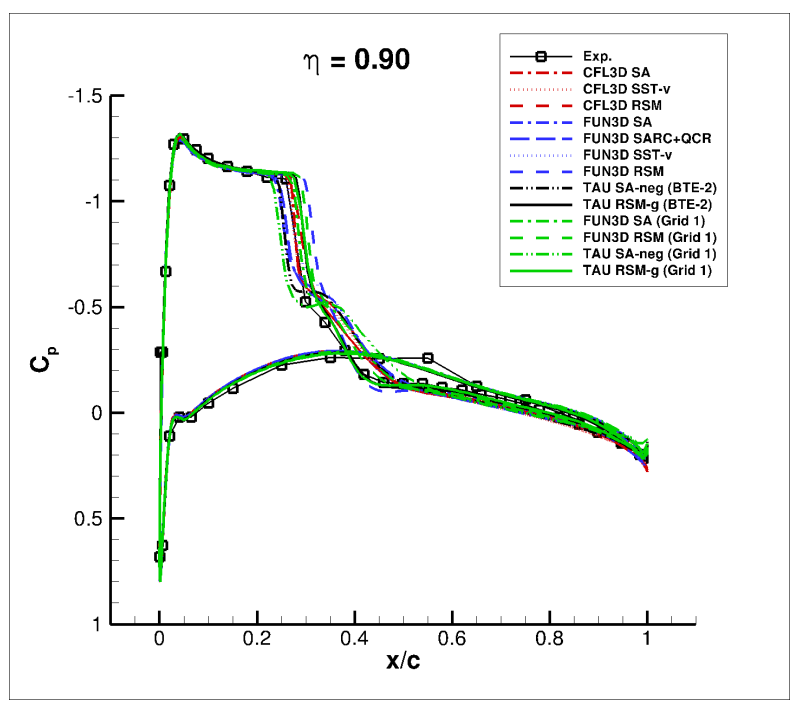

(a) All codes, models and grids

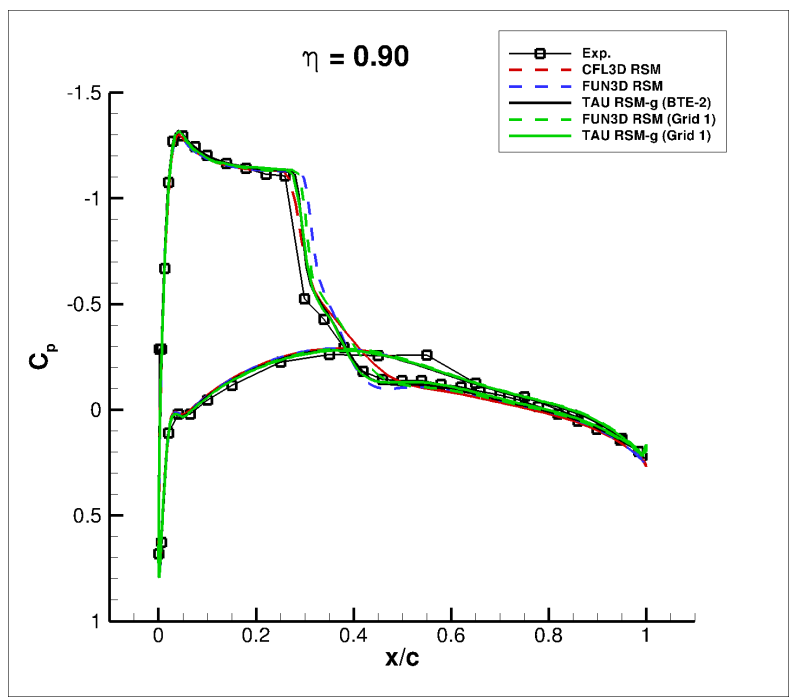

(c) RSM models

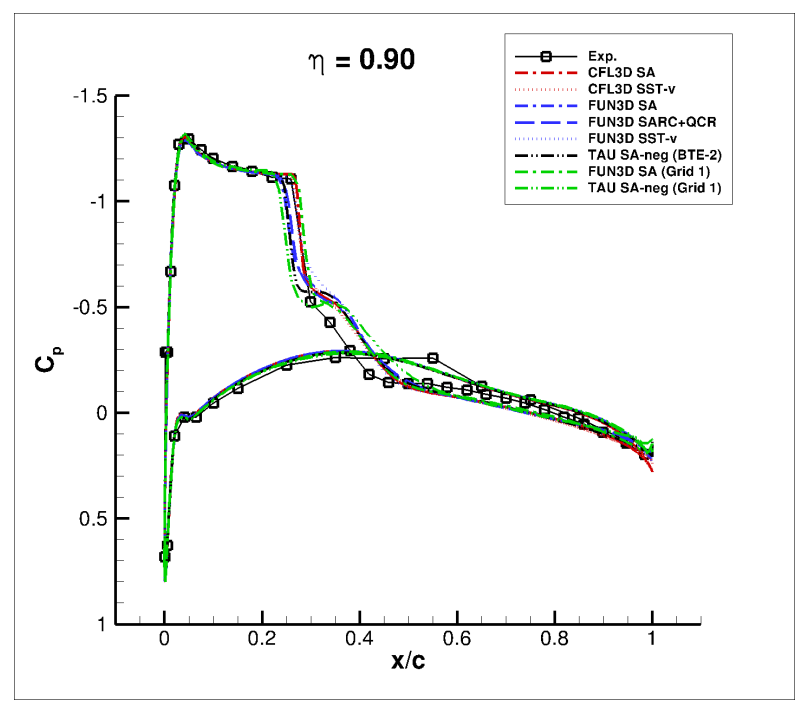

(b) One and two equation models

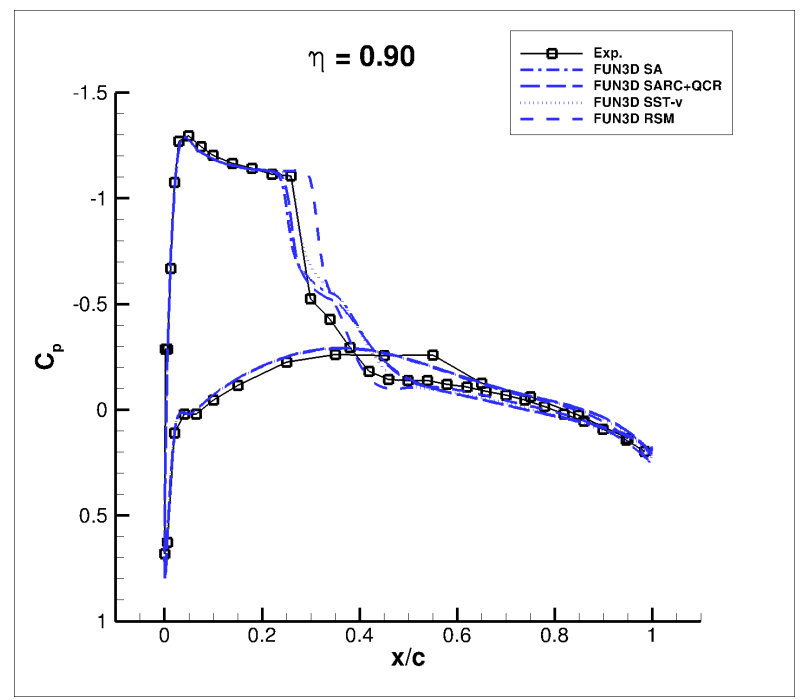

(d) All models, one code and one grid

Figure 4. Comparison of ONERA M6 sectional pressure coefficient at $90 \%$ span station. 


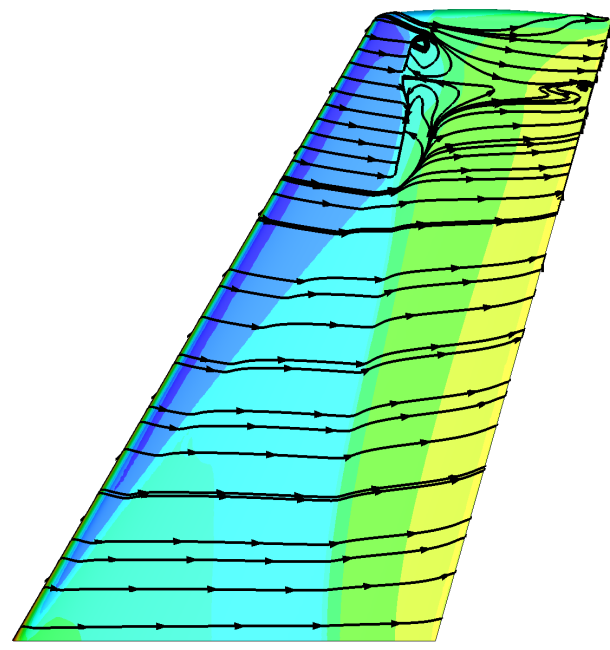

(a) TAU, SA-neg, Grid 1

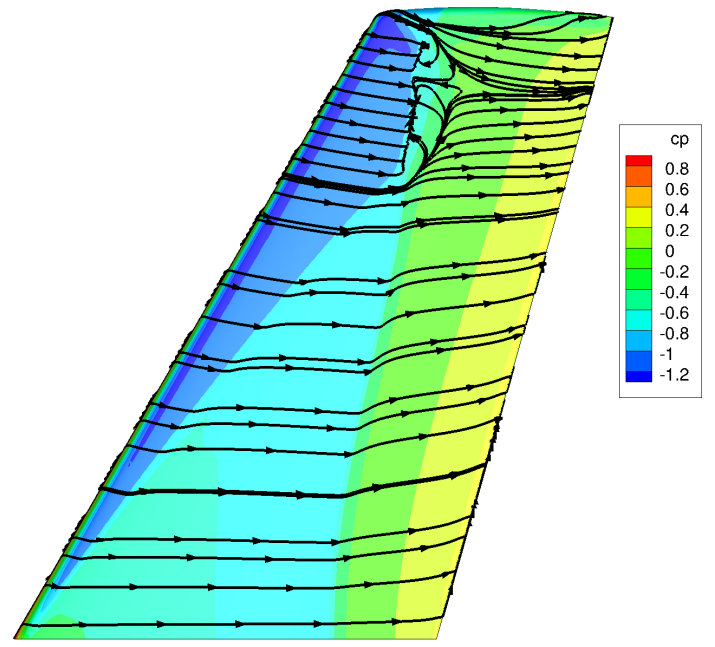

(c) FUN3D, SA, Grid 1

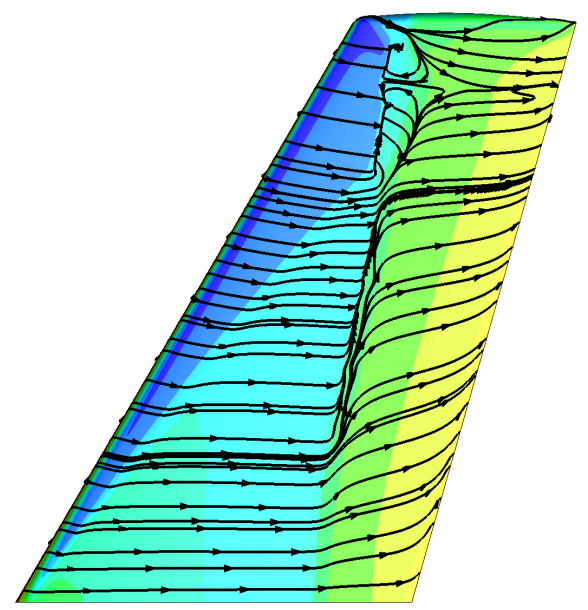

(e) FUN3D, SA, unstructured baseline grid

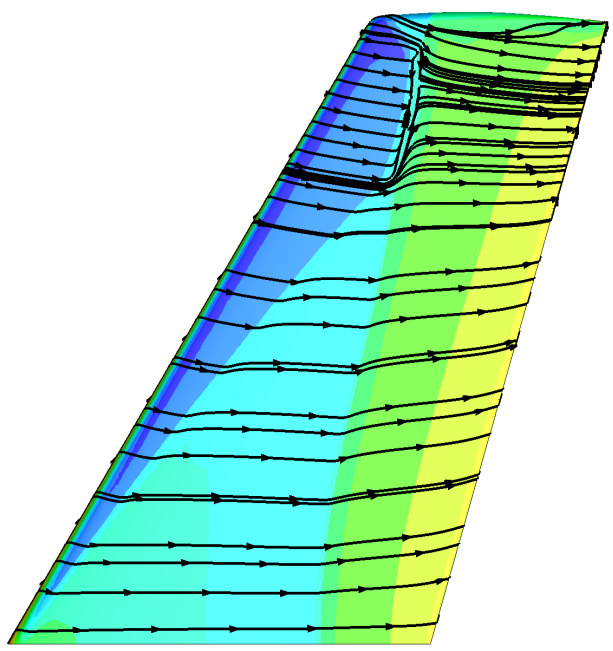

(b) TAU, RSM-g, Grid 1

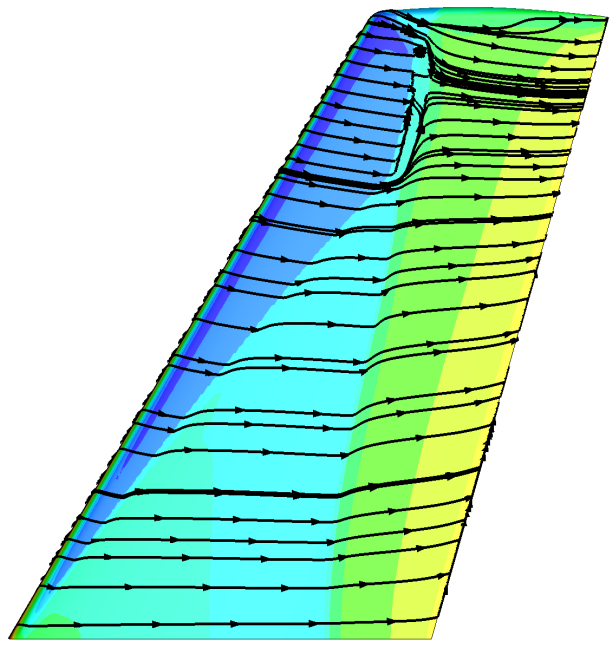

(d) FUN3D, RSM, Grid 1

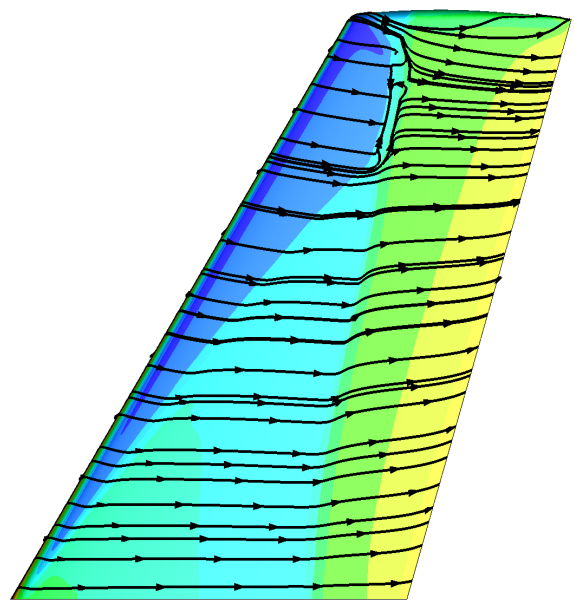

(f) FUN3D, RSM, unstructured baseline grid

Figure 5. Comparison of ONERA M6 surface restricted streamlines and $C_{p}$. 
quantities for the DLR-F11 are summarized in Table 1.

Table 1. Reference geometry for the DLR-F11.

$\begin{array}{ll}\text { Wing Reference Chord, } c_{r e f} & 0.34709 \text { meter } \\ \text { Wing Reference Area, } A / 2 & 0.41913 \text { meter }^{2} \\ \text { Wing Half Span, } b / 2 & 1.4 \text { meter } \\ \text { Aspect Ratio, } A R & 9.353 \\ \text { Quarter Chord Sweep angle } & 30.0^{\circ}\end{array}$

For the workshop CFD simulations, the geometry was approximated in a series of configurations that built up the model for improved geometric fidelity:

- Config 2 - wing/body/high lift (HL) system plus side-of-body (SOB) flap seal;

- Config 4 - same as Config 2 plus slat and flap support brackets;

- Config 5 - same as Config 4 plus slat pressure tube bundles

The model has seven slat support brackets/fairings (SB1-SB7) and five flap support brackets/fairings (FB1FB5). These are numbered from inboard to outboard. Figure 6 shows the location of the experimental pressure measurement stations in relation to the slat and flap brackets for later reference. Oil visualization from the low Reynolds number experiment suggests that at stall large isolated areas of separated flow exist over the main wing behind two of the slat brackets (SB5 and SB6) and in front of two of the flap tracks (FB3 and FB4), which spread out laterally toward the trailing edge. Although oil flow visualization was not performed during the high Reynolds number test, the pressure measurements and force/moment trends suggest that a similar stall mechanism takes place at the high Reynolds number condition.

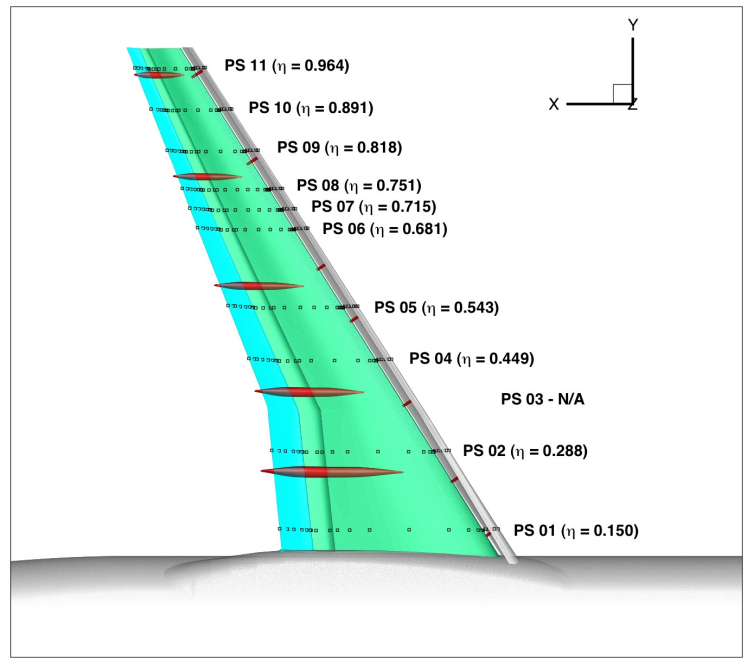

Figure 6. DLR F11 experimental pressure stations in relation to the slat and flap brackets.

While the wind tunnel model was tested as a semi-span model, all the workshop test cases were specified to be run in "free-air" with a computational symmetry plane at the center line of the geometry. The experiment did not have specified transition on the model. However, in the workshop, the boundary layer was to be modeled as "fully turbulent" in the CFD simulations, i.e., turbulence is modeled over the entire configuration. Details regarding the experimental geometry, data and repeatability can be found in Rudnik et al. $^{33}$ 


\section{V.B. Computational Meshes}

The approach for this RSM study is to build upon the prior FUN3D analysis of the HiLiftPW-2 test cases. Lee-Rausch et al. ${ }^{11}$ presented results from FUN3D with the SA and SA-RC models on workshop-provided grids (Grid System D), as well as on adapted grids. For the workshop, grid refinement studies were performed on the simplified Config 2 and only medium-size grids based on "best-practices" were generated for Configs 4 and 5 . In the current study, no grid adaption was performed, and only the medium-level grids were analyzed. However, the additional geometric complexity of the support brackets and pressure tube bundles is believed to be important, as these components were found during HiLiftPW-2 to be influential near maximum lift.

Here, two different grid systems from the workshop are considered. Grid System C grids were built by Pointwise Inc. with the Pointwise $\AA$ software and have a combination of prisms in the near-wall region and tetrahedra in the rest of domain. Grid System D grids were built by the University of Wyoming and Cessna with VGRID v4.0 and have a combination of prisms in the near-wall region and tetrahedra in the rest of domain. ${ }^{34}$ A summary of the grid sizes is given in Table 2. The target wall spacing was $y^{+} \sim 2 / 3$ for the high Reynolds number condition; the same mesh was used for Grid Systems C and D at both the low and high Reynolds number conditions.

Table 2. Comparison of medium-grid sizes (in millions of nodes) for workshop-supplied grids.

\begin{tabular}{lll} 
Configuration & Grid System C & Grid System D \\
\hline Config 2 & 57 & 31 \\
Config 4 & 65 & 42 \\
Config 5 & & 44
\end{tabular}

\section{V.C. Summary of Cases}

As previously mentioned, computations with FUN3D were made on two different grid systems from the workshop. However, due to robustness issues with the solver, no RSM computations were completed with the Grid System C meshes. FUN3D computations were made with the RSM model only for the Grid System $\mathrm{D}$ meshes at both low and high Reynolds numbers. In order to compare the RSM model with the most up-to-date SA model corrections, additional FUN3D computations with the SA-RC-QCR2000 model were completed for the Grid System D meshes at both low and high Reynolds numbers. In an attempt to provide some assessment of the influence of grid, FUN3D computations with the SA-RC-QCR2000 model were also made for the Grid System C Config 4 mesh.

Many workshop participants in the first high-lift prediction workshop noted a sensitivity of their solutions to the initial conditions of the computation, especially at higher angles of attack. ${ }^{35}$ The first and second authors also found that initializing the computation with free-stream conditions could result in a solution with stall characteristics at lower angles of attack than a solution that was initialized from a previously converged computation at a lower angle of attack. ${ }^{36}$ Therefore in the current computations, solutions were initialized from a previously converged computation at a lower angle of attack (when possible).

\section{V.D. Force and Moment Comparisons}

FUN3D results for the SSG/LRR-RSM-w2012-SD model at the high Reynolds number condition $\left(R e_{C}=15.1\right.$ million) are shown in Fig. 7 for the Grid System D meshes. Here, "RSM" is used in the figure as shorthand notation for the SSG/LRR-RSM-w2012-SD model. Comparisons are made with experimental data and the original SA computations from the workshop. At angles of attack $\alpha \leq 16^{\circ}$, the SSG/LRR-RSM-w2012-SD model results tended to yield slightly higher lift, in better agreement with experiment than SA. Near $C_{L, \max }$, the RSM tended to overpredict lift regardless of the presence of the brackets and/or pressure tube bundles; these features only had a slight mitigating influence. On the other hand, the SA model overpredicted $C_{L, \max }$ for Config 2 only.

The presence of brackets and pressure tube bundles in Configs 4 and 5 caused the SA model to separate on the main element too early, yielding values of lift at and above $\alpha=18.5^{\circ}$ below those measured. LeeRausch et al. ${ }^{11}$ showed that SA model results could be improved near $C_{L, \max }$ with adaptive mesh refinement. 
However, adjoint-based mesh refinement for SSG/LRR-RSM-w2012-SD could not be completed for this study as this model has not been implemented in the FUN3D adjoint solver.

Both SA and RSM models yielded similar comparison with drag experimental data, tending to be somewhat too high at the lower angles of attack and significantly off near $C_{L, \max }$ conditions. And both models significantly underpredicted moment levels, with SA somewhat closer to experiment than the RSM. As conjectured in Ref. 11, differences in drag and moment levels between CFD and experiment may be due to the effect of semi-span testing.

The Config 4 polars from Fig. 7 are further compared to SA-RC-QCR2000 results with Grid System C and D meshes in Fig. 8. In this comparison, the SA-RC-QCR2000 results on Grid D are now in much closer agreement with the SSG/LRR-RSM-w2012-SD results. Similarly, the SA-RC-QCR2000 results on Grid C are also in close agreement with the Grid D SA-RC-QCR2000 and SSG/LRR-RSM-w2012-SD results. The SA-RC-QCR2000 and RSM results all overpredict the lift near $C_{L, \max }$, but they do stall somewhere between $\alpha=22^{\circ}$ and $24^{\circ}$ (not shown). The solutions at $\alpha=24^{\circ}$ have large amounts of separated flow on the main element and are difficult to converge.

Similar plots of the Config 4 polars for the low Reynolds number $\left(R e_{C}=1.35\right.$ million $)$ condition are shown in Fig. 9. There is a much larger spread in results near maximum lift than was seen at the higher Reynolds number. The RC and QCR corrections in the SA model do prevent early stall on the D Grid at $\alpha=18.5^{\circ}$, but the lift and drag are still somewhat overpredicted. The RSM results on the D Grid compare more closely with the experimental lift and drag near $C_{L, \text { max }}$, but the pitching moment is still overpredicted (too negative) at maximum lift. The SA-RC-QCR2000 results on Grid C compare well with the Grid D results up to $\alpha=19^{\circ}$, but the lift does not drop until $\alpha=21^{\circ}$.

\section{V.E. Surface Flow Comparisons}

Comparisons of surface-restricted streamlines (black) and streamwise skin friction contours are shown in Fig. 10 for Config 4 Grid D computations at the high Reynolds number condition. The locations of the experimental surface pressure span stations are noted with white lines for comparisons with subsequent surface pressure plots. All models yielded very similar results at $\alpha=18.5^{\circ}$; but at $\alpha=20^{\circ}$, the SA model separates on the main element behind the outboard slat bracket SB6 while the SA-RC-QCR2000 and RSM remained attached at $\alpha=22^{\circ}$ where these models overpredict the lift in comparison with the experimental data (see Fig. 8). The SA-RC-QCR2000 model shows a very small area of separation behind SB6 at $\alpha=22^{\circ}$ near the trailing edge of the main element. Grid refinement for the SA-RC-QCR2000 model does not impact the high-lift prediction in that the SA-RC-QCR2000 results for Grid C (not shown) show a similar pattern of surface flow for this case at $\alpha=18.5^{\circ}$ and $22^{\circ}$. None of the models and grids seem to be accurately predicting the more gradual growth of flow separation with increased angle of attack that is indicated in the overall force and moment experimental data. Experimental oil flow visualizations are not available at the high Reynolds number to verify the flow separation patterns.

Oil flow visualizations are available at the low Reynolds number condition. Surface-restricted streamlines and streamwise skin friction contours are shown in Fig. 11 for the Config 4 Grid D computations and are compared with the experimental oil flows. At $\alpha=18.5^{\circ}$, the oil flow patterns indicate small areas of wedgeshaped separation near the main element trailing edge behind both SB5 and SB6. In Fig. 11(c) and (e), the SA-RC-QCR2000 and RSM computations do not show these separation patterns at $\alpha=18.5^{\circ}$ in Fig. 11(c) and (e). At $\alpha=21^{\circ}$, the oil flow patterns indicate a much larger area of wedge-shaped separation on the main element behind only SB5. By $\alpha=20^{\circ}$, the SA-RC-QCR2000 and RSM computations show a large area of wedge-shaped separation on the main element, but it is behind SB6 and no separation is indicated behind SB5. By $\alpha=21^{\circ}$ (not shown), the computed separation has spread across the outboard portions of the slat and main element. As previously shown in Ref. 11, the SA computations on the D Grid predict a large area of wedge-shaped separation on the main element behind SB6 as early as $\alpha=18.5^{\circ}$.

Grid refinement for the SA-RC-QCR2000 model does show an impact for the low Reynolds number case at the higher angles of attack. Surface-restricted streamlines for Grid C are shown in Fig. 12 and are compared with Grid D results and the experimental oil flows. At $\alpha=18.5^{\circ}$, Grid $\mathrm{C}$ results also do not show the small wedge-shaped patterns of separation on the main element. However, at $\alpha=21^{\circ}$, the Grid $\mathrm{C}$ results predict a much smaller area of separation than Grid D predicts. The size of the predicted separation on Grid C is more comparable to the size of the separation shown in the experimental oil flow. But, as with the Grid D computations, the separation is predicted behind SB6 and not behind SB5 as seen in the experimental oil flow. No computed separation occurs behind SB5. 


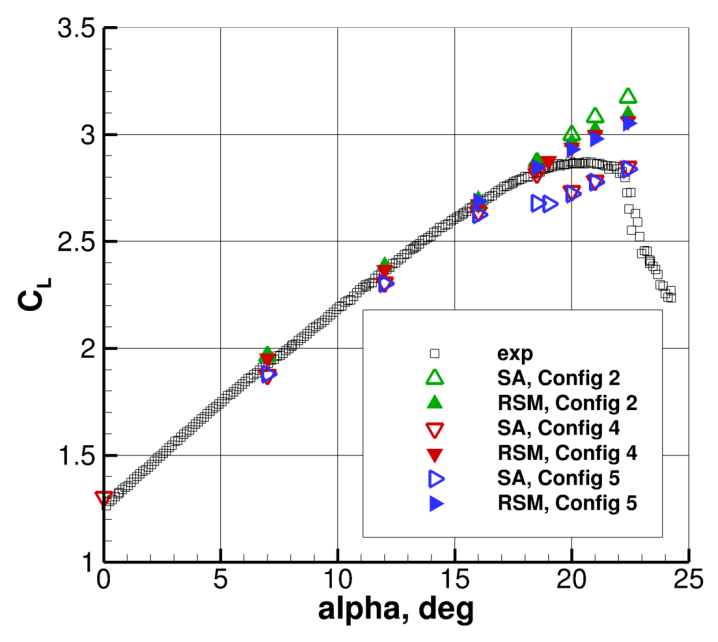

(a) Lift vs. alpha

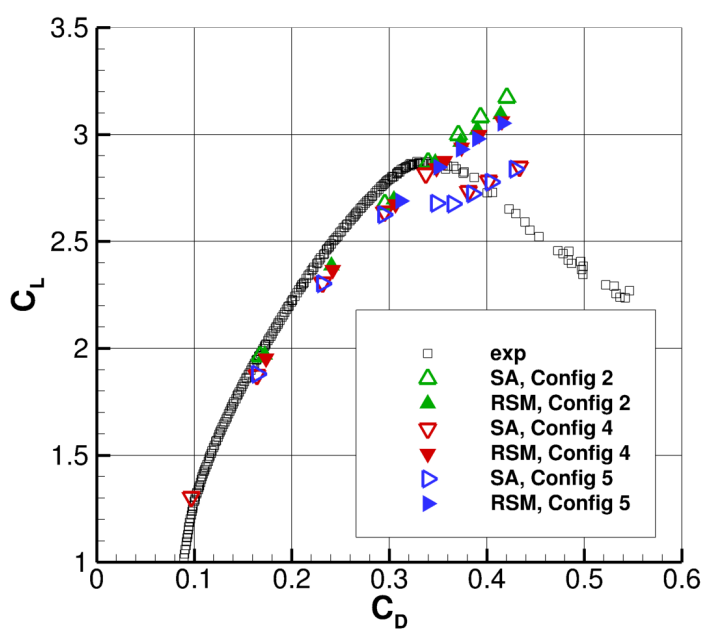

(b) Lift vs. drag

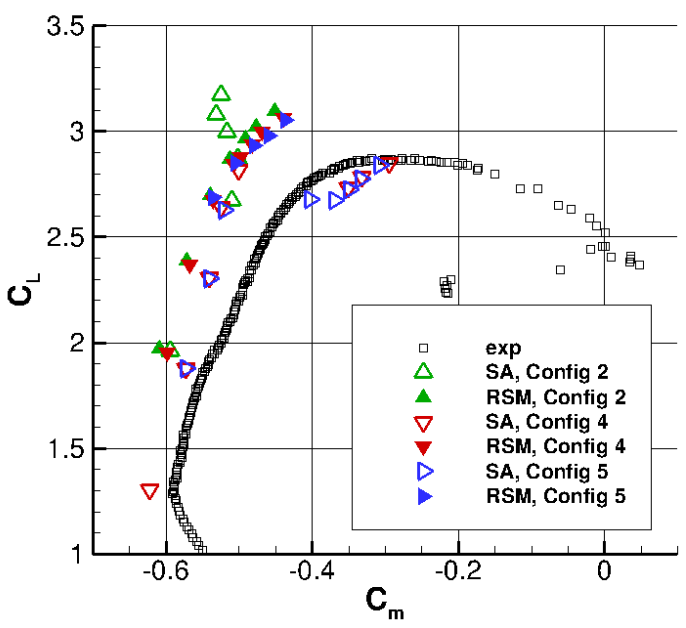

(c) Lift vs. pitching moment

Figure 7. FUN3D force and moment polars for DLR-F11 at high Reynolds number conditions.

In Fig. 9, the RSM Grid D computations seem to be predicting the gradual onset of stall that is indicated by the low Reynolds number force and moment experimental data. However, comparisons with experimental surface flow patterns in Fig. 11 show that the RSM computations fail to predict the gradual growth of separation on the main element behind the outboard slat brackets at the lower angles of attack. For all the models, the flow stays attached on the main element behind the brackets at the lower angles of attack resulting in an overprediction of lift, drag and (negative) pitching moment. As the angle of attack is increased, the main element separation is more abrupt in the computations, and it occurs behind the wrong slat bracket. Some sensitivity to grid refinement (Grid C versus Grid D) was shown for the SA-RC-QCR2000 model, but grid refinement effects were not explored for the RSM. Potentially, the inclusion of the pressure tube bundles could influence the maximum lift predictions at the low Reynolds number condition. This should be considered in a future study.

\section{V.F. Surface Pressure Comparisons}

Comparisons of chordwise surface pressures are shown in Figs. 13 and 14 for Config 4 computations at the high Reynolds number condition. Results for the SA-RC-QCR2000 and RSM computations are compared with 
the experimental data across the span at $\alpha=18.5^{\circ}$ and $22^{\circ}$. Note the SA model results were not included because it was clear from the comparisons of the polars and surface flows that the flow had separated early at these angles of attack in comparison with the other models and experiment. At $\alpha=18.5^{\circ}$, the variations in the computed surface pressures are very small and primarily occur on the flap at the root and tip. (Note the vertical scale is expanded at the root and tip stations.) The overall comparison with the experimental data is very good. The overprediction of lift and (negative) pitching moment shown in Fig. 8 could be attributed to the overprediction of suction pressures on the flap across the span. Comparisons of main element surface pressures nearest to SB5 $(\eta=0.54)$ and SB6 $(\eta=0.75)$ show that there is no significant variation between any of the models and grids. The computed results are closer to the experiment at $\eta=0.54$ but show some overprediction of suction pressure near the trailing edge of the main element and leading edge of the flap possibly at $\eta=0.75$, indicating that in the experiment some separation had started to occur in this area which is not predicted in the computations.

At $\alpha=22^{\circ}$, the variations in the computed surface pressures are more significant and primarily occur on the main and flap elements between $\eta=0.72$ and $\eta=0.82$. However, the Grid D RSM and the Grid C SA-RC-QCR2000 pressure overlay in all plots except the tip. The overall comparison with the experimental data is poor with overprediction of the suction pressures on all elements on the outboard wing. Even without oil flow data, the experimental pressures indicate that large amounts of separation form on the main element outboard of $\eta=0.54$ that is not being predicted by any of the models, which correlates with the overprediction of lift and (negative) pitching moment shown in Fig. 8.

Similar comparisons of chordwise surface pressures are shown in Figs. 15 and 16 for Config 4 computations at the low Reynolds number condition. Results for the SA-RC-QCR2000 and RSM computations are compared with the experimental data across the span at $\alpha=18.5^{\circ}$ and $20^{\circ}$. At $\alpha=18.5^{\circ}$, the variations in the computed surface pressures are very small and primarily occur at the root and tip. (Note the vertical scale is expanded at the root and tip stations.) The overall comparison with the experimental data is very good inboard but some overprediction of suction pressures are shown for all computations outboard of $\eta=0.45$, which correspond with the overprediction of lift and negative pitching moment in Fig. 9. By $\alpha=20^{\circ}$, the variations in the computations is more significant and mainly occurs outboard of $\eta=0.54$. The Grid $\mathrm{D}$ RSM and SA-RC-QCR2000 computations predict lower suction pressures than the Grid C SA-RC-QCR2000 computations and the experiment near SB6 $(\eta=0.75$ and $\eta=0.82)$. The Grid D computations in Fig. 9 are closer to the experimental force and moment measurements at $\alpha=20^{\circ}$, but clearly the pressures prediction are much farther from the measurement, probably due to some compensating effect. There is a much clearer influence of grid refinement (Grid C versus Grid D) for the SA-RC-QCR2000 predicted pressures near maximum lift than was seen at the higher Reynolds number condition; but grid refined RSM computations need to be performed before a more definitive assessment of the model can be made.

\section{Summary and Future Research}

A recently developed second-moment Reynolds stress model from DLR was applied to two challenging high-lift flows: (1) transonic flow over the ONERA M6 wing, and (2) subsonic flow over the DLR-F11 wing-body configuration from the second AIAA High Lift Prediction Workshop. The Reynolds stress model computational results were contrasted with those obtained from other simpler turbulence models. Multiple codes, grids and turbulence models were used to compute transonic, high angle-of-attack solutions for the ONERA M6 wing. At most span stations, the range of results showed only relatively small variations from each other, with little difference between RSM and other models. However, at the $90 \%$ span station, the range of results showed larger variation in the prediction of the shock location and shock-induced separation. The one- and two-equation model predictions showed slightly larger areas of shock-induced separation than the RSM predictions, but all models were fairly close to the limited experimental surface pressure data, making it difficult to draw firm conclusions regarding model fidelity. A previously reported significant overprediction of shock-induced separation by the SA and SST models was not repeated in the current study. However, there is some indication of a multi-valued solution with TAU using SA-neg on Grid 1. This possibility remains an open question.

The HiLiftPW-2 test cases included both high and low Reynolds number wind tunnel conditions. The workshop CFD geometry included three configurations that built up the model complexity for increased geometric fidelity, with the most complete configurations including support brackets and pressure tube bundles. Several grid systems were available from the workshop for the current study that included the more 
complete geometric representation. FUN3D computations were performed on the three configurations from the Grid System D meshes at the high and low Reynolds number conditions. However, due to robustness issues with the solver, no FUN3D RSM computations were completed with the Grid System C meshes. For comparison of the RSM model with the most up-to-date SA model corrections, additional FUN3D computations with the SA-RC-QCR2000 model were completed for the Grid System D meshes at both high and low Reynolds numbers. FUN3D computations with the SA-RC-QCR2000 model were also computed for the Grid System C Config 4 mesh.

For the high Reynolds number condition, the RSM and SA-RC-QCR2000 models overpredicted the maximum lift in comparison with the experiment, in contrast with the SA model that stalled early for all but the simplest geometry. A detailed comparison of surface flows patterns and pressure measurements indicated that none of the models and grids in the current study seem to be accurately predicting the growth of flow separation on the main element with increased angle of attack that is indicated in the experimental data. For the low Reynolds number condition, the RSM and SA-RC-QCR2000 models also overpredicted the maximum lift in comparison with the experiment, while the SA model again stalled early. The amount of overprediction was smaller than for the high Reynolds number conditions, and there was a wider variation between the RSM and SA-RC-QCR2000 results near stall. Although the RSM model maximum lift prediction was close to the experimental data, the surface pressures for both the RSM and the SA-RC-QCR2000 models indicated more separation on the main wing than was present in the experimental data.

Overall, the SSG/LRR-RSM-w2012 model was competitive with the one- and two-equation models in terms of the prediction of shock location and separation. For the ONERA M6 case, results from multiple codes, grids and models were compared. However, for the DLR-F11 cases, the range of RSM results were limited to one code and one grid system. Additional computations need to be performed before a more definitive assessment of the RSM model can be made. Current efforts are underway to improve the iterative

convergence and solver robustness for the RSM in FUN3D. Results from this study will provide a basis of comparison for future DLR-F11 RSM computations.

\section{Acknowledgment}

The first and second authors' work was performed as part of the Revolutionary Computational Aerosciences discipline under the Transformational Tools and Technologies project of NASA's Transformative Aeronautics Concepts Program. 


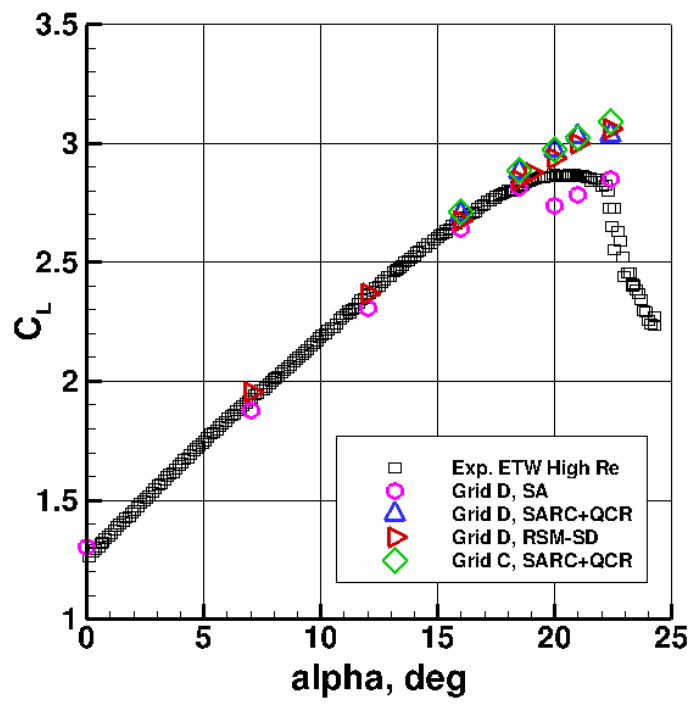

(a) Lift vs. alpha

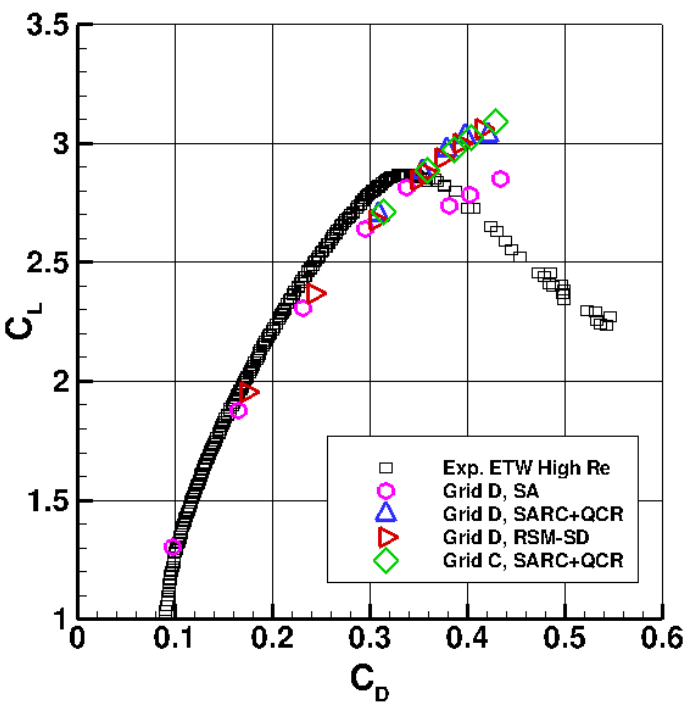

(b) Lift vs. drag

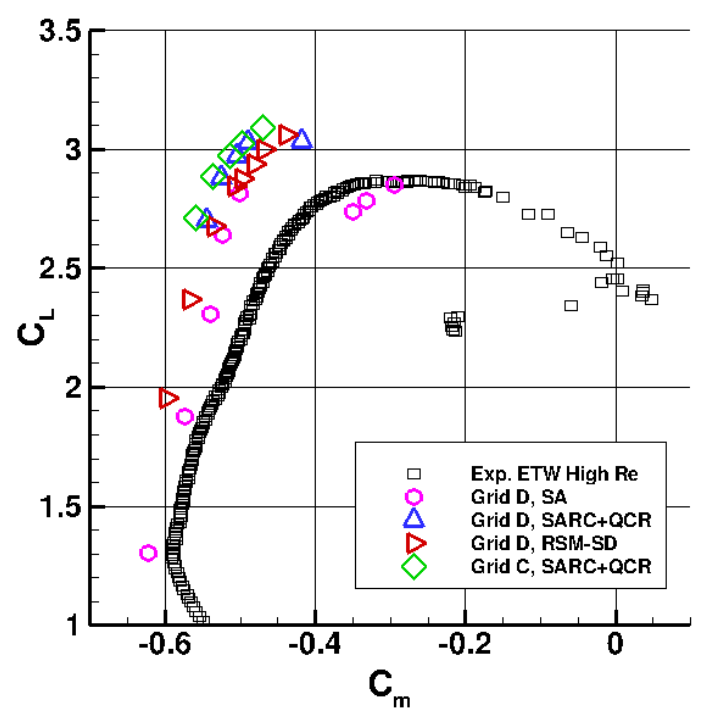

(c) Lift vs. pitching moment

Figure 8. FUN3D force and moment polars for Config 4 at high Reynolds number conditions. 


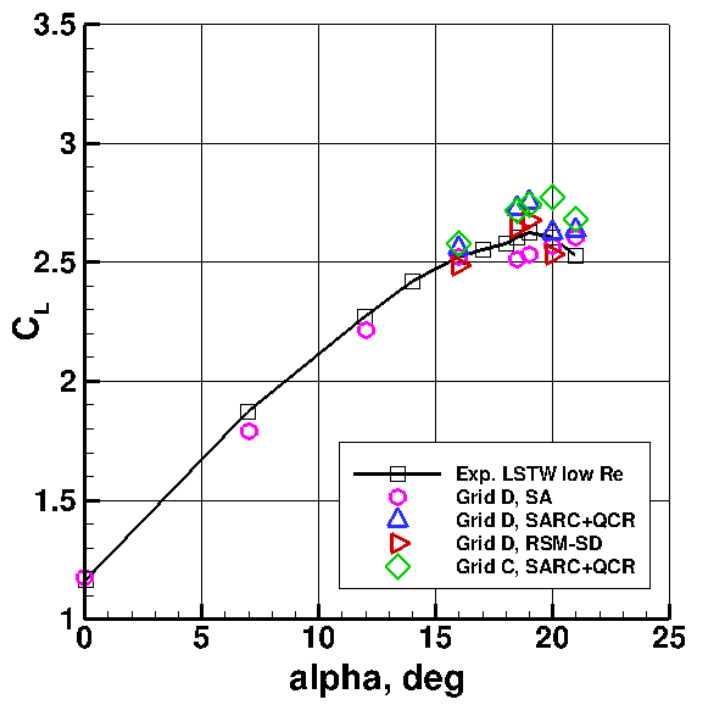

(a) Lift vs. alpha

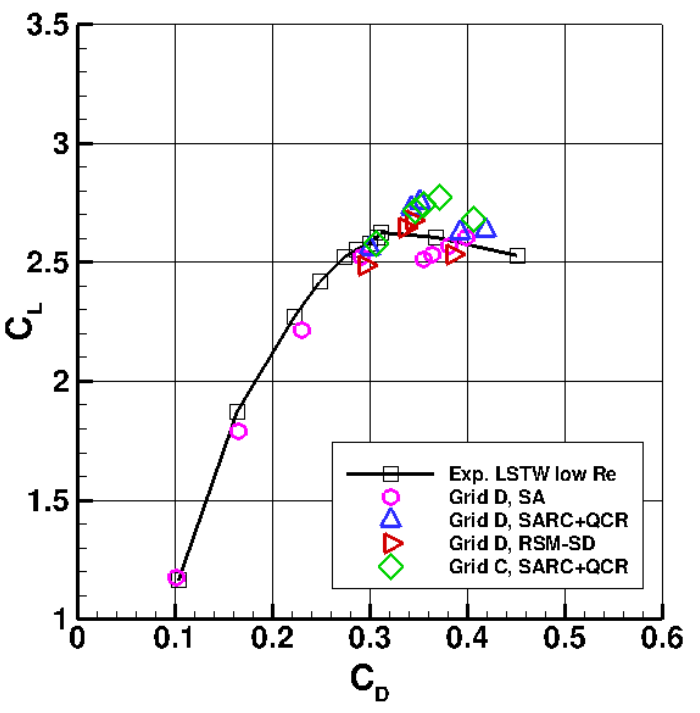

(b) Lift vs. drag

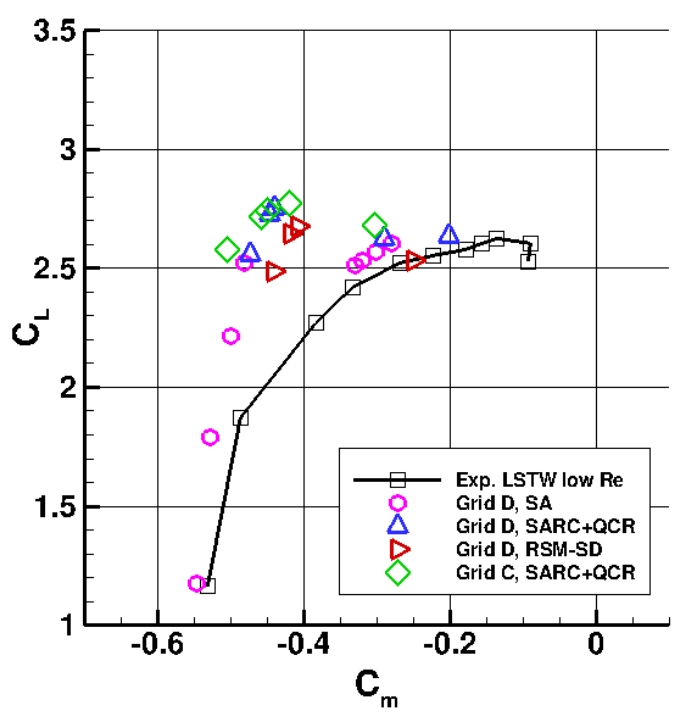

(c) Lift vs. pitching moment

Figure 9. FUN3D force and moment polars for Config 4 at low Reynolds number conditions.

\section{9 of 28}




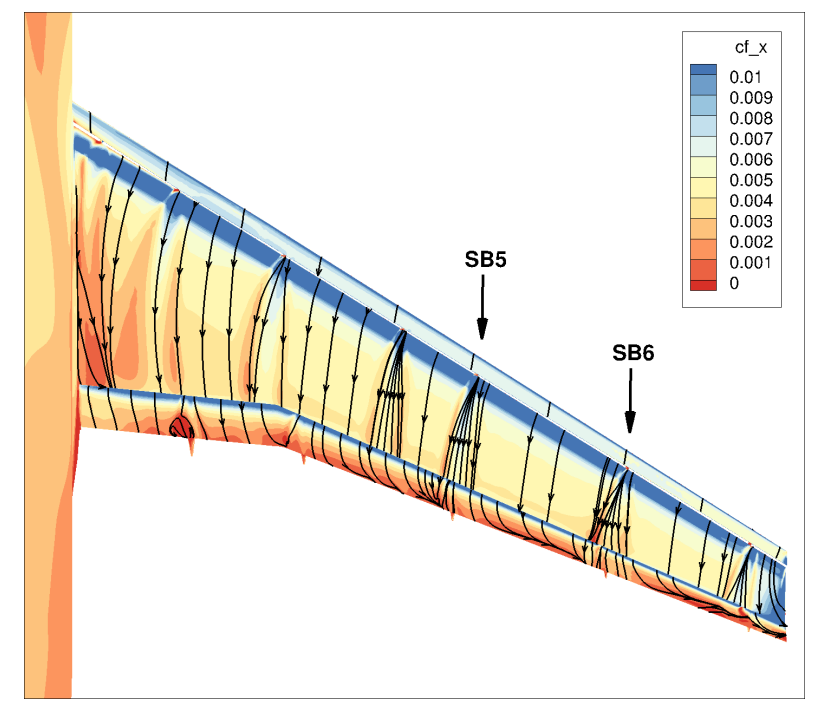

(a) $\mathbf{S A}, \alpha=18.5^{\circ}$

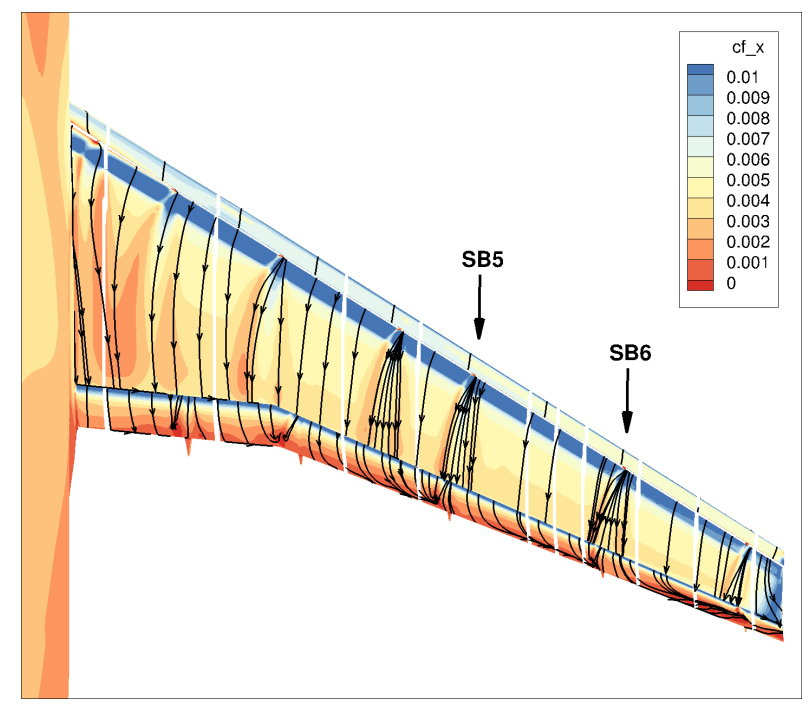

(c) SA-RC-QCR2000, $\alpha=18.5^{\circ}$

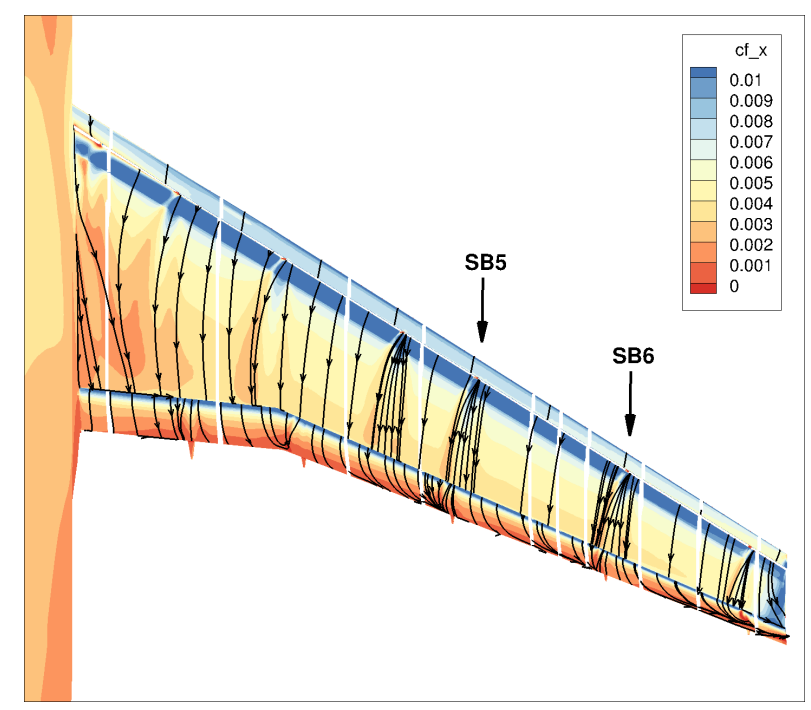

(e) RSM, $\alpha=18.5^{\circ}$

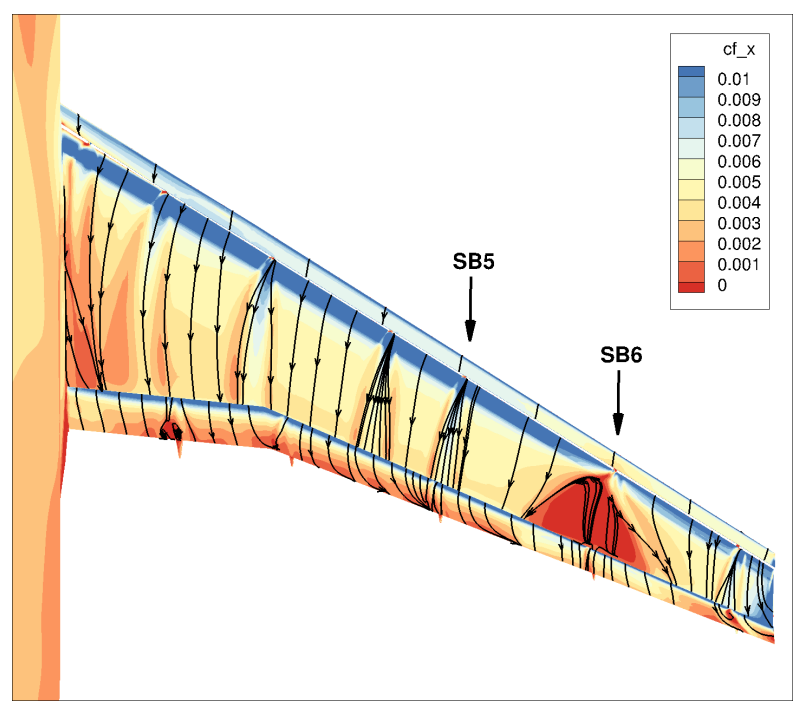

(b) $\mathbf{S A}, \alpha=20.0^{\circ}$

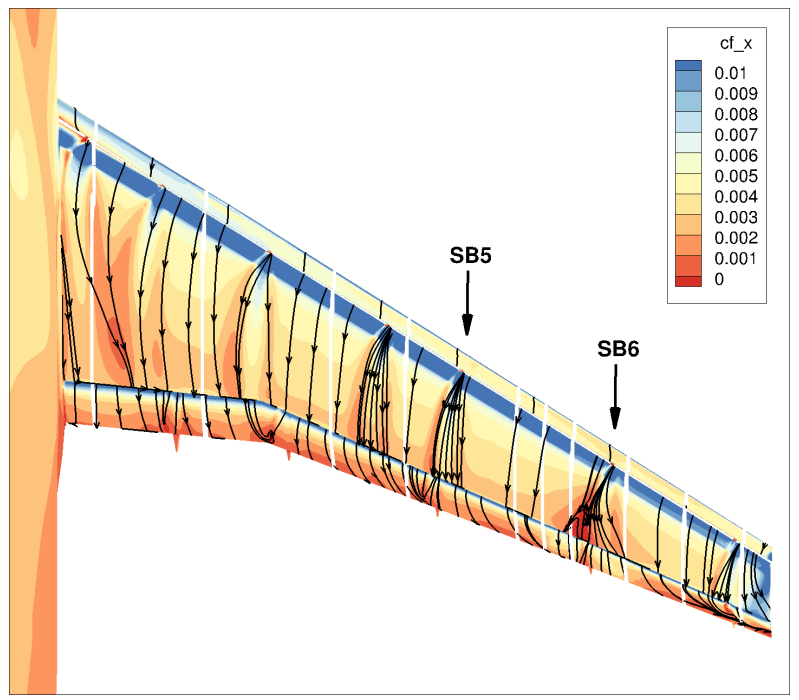

(d) SA-RC-QCR2000, $\alpha=22.0^{\circ}$

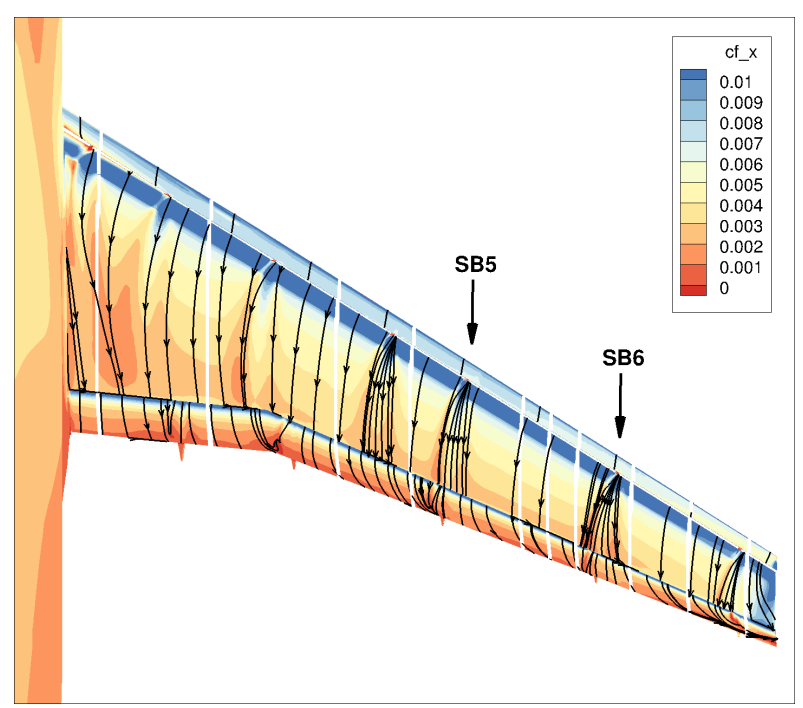

(f) $\operatorname{RSM}, \alpha=22.0^{\circ}$

Figure 10. Comparison of high $R_{C}$ Config 4 surface restricted streamlines and $C_{f}$ near stall (white lines pressure measurement stations). 


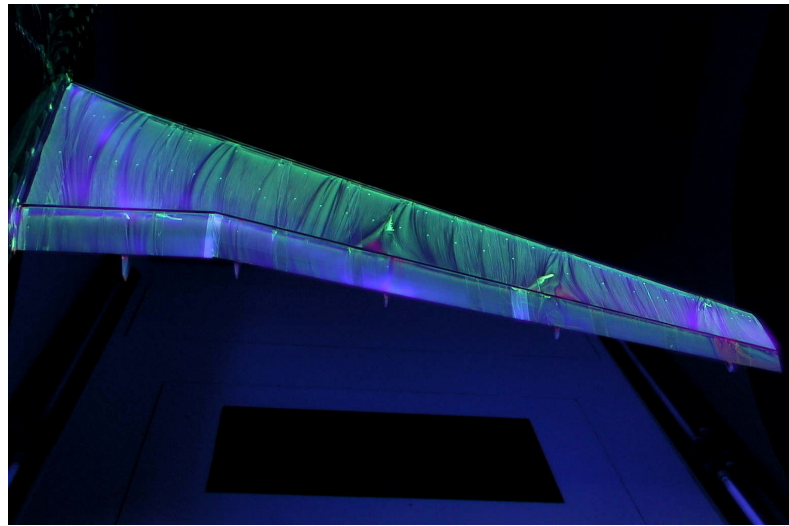

(a) Exp., $\alpha=18.5^{\circ}$

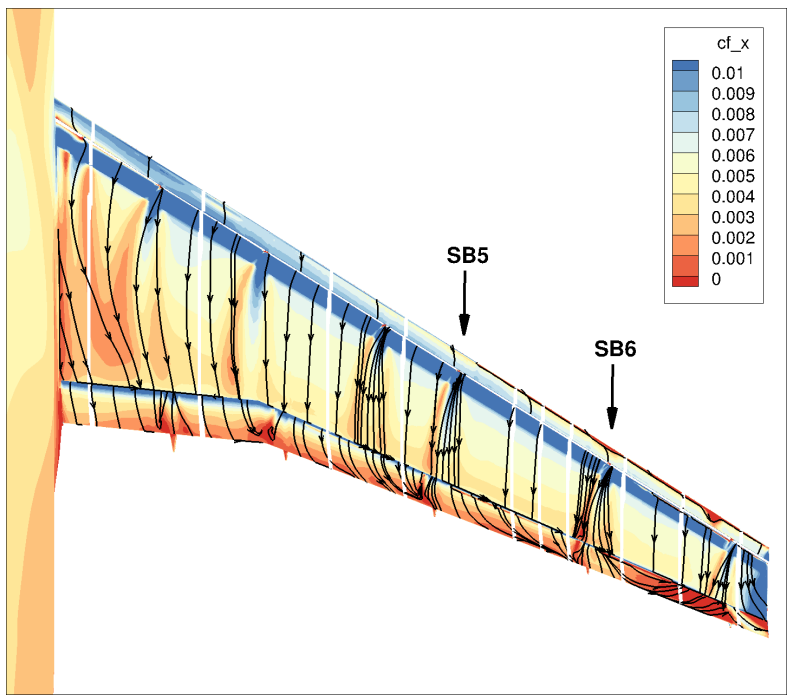

(c) SA-RC-QCR2000, $\alpha=18.5^{\circ}$

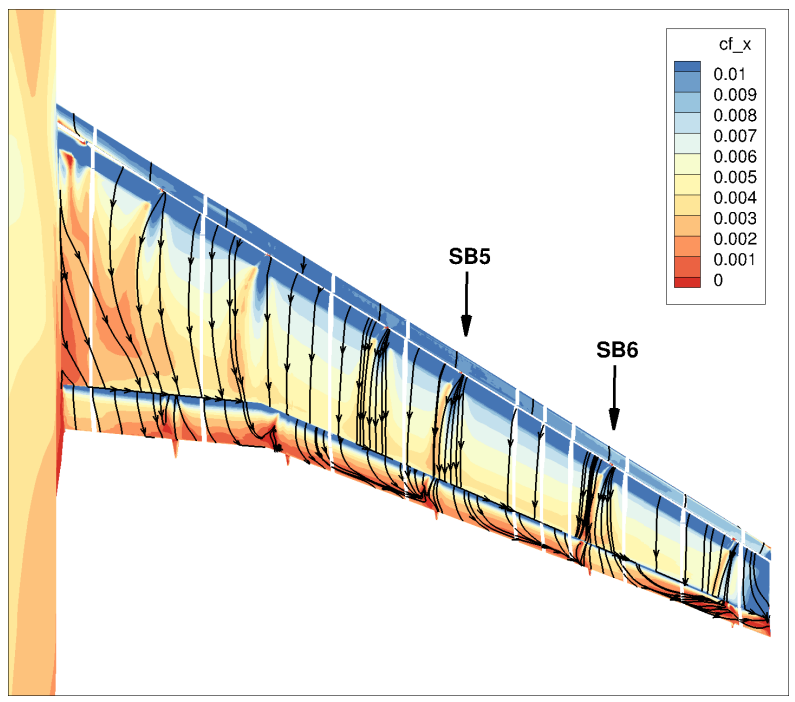

(e) RSM, $\alpha=18.5^{\circ}$

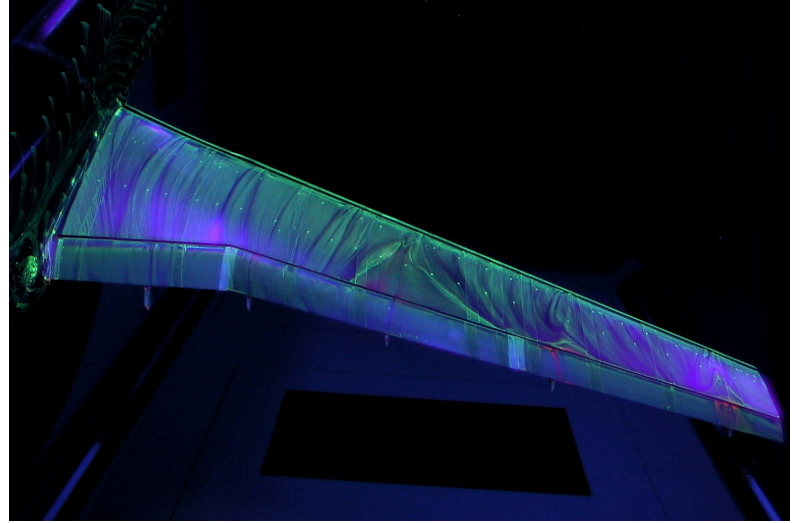

(b) Exp., $\alpha=21.0^{\circ}$

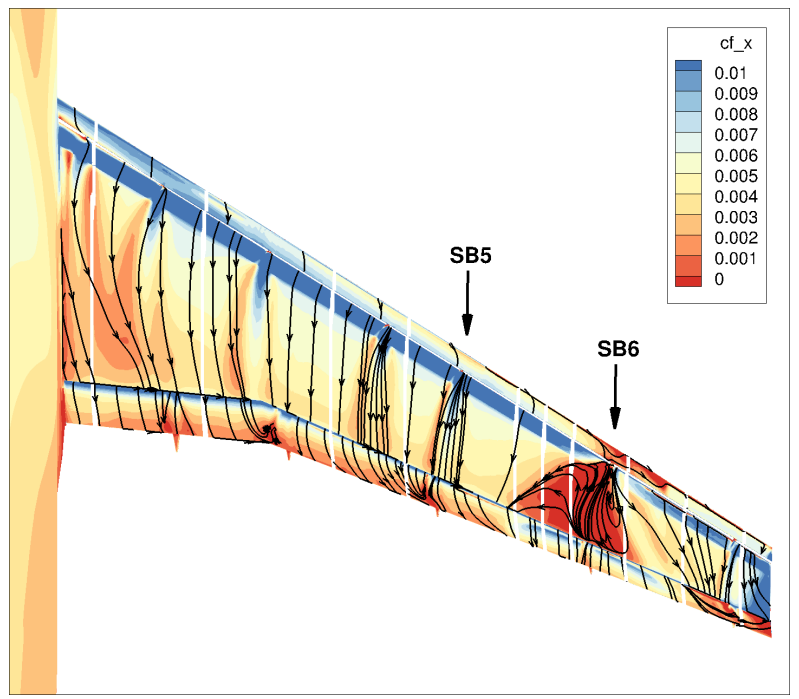

(d) SA-RC-QCR2000, $\alpha=20.0^{\circ}$

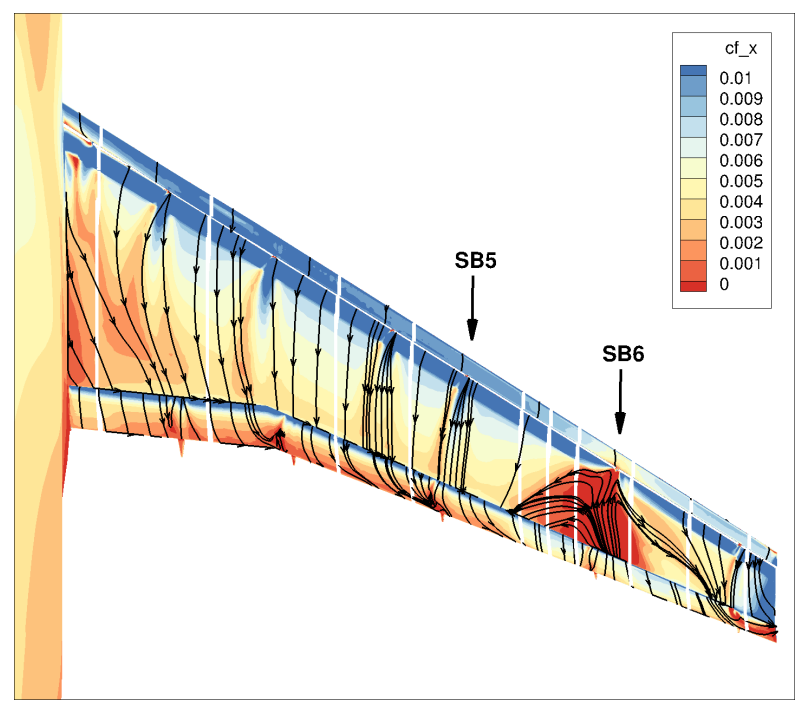

(f) RSM, $\alpha=20.0^{\circ}$

Figure 11. Comparison of low $\operatorname{Re}_{C}$ Config 4 surface restricted streamlines and $C_{f}$ near stall (white lines pressure measurement stations). 


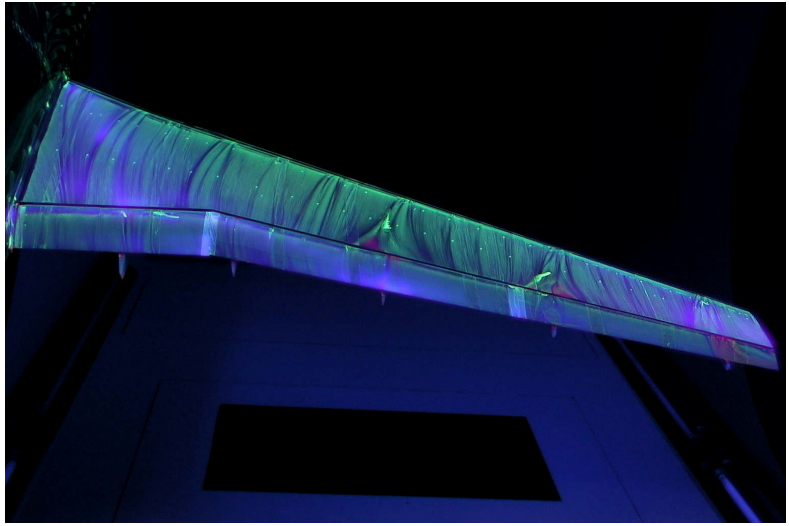

(a) Exp., $\alpha=18.5^{\circ}$

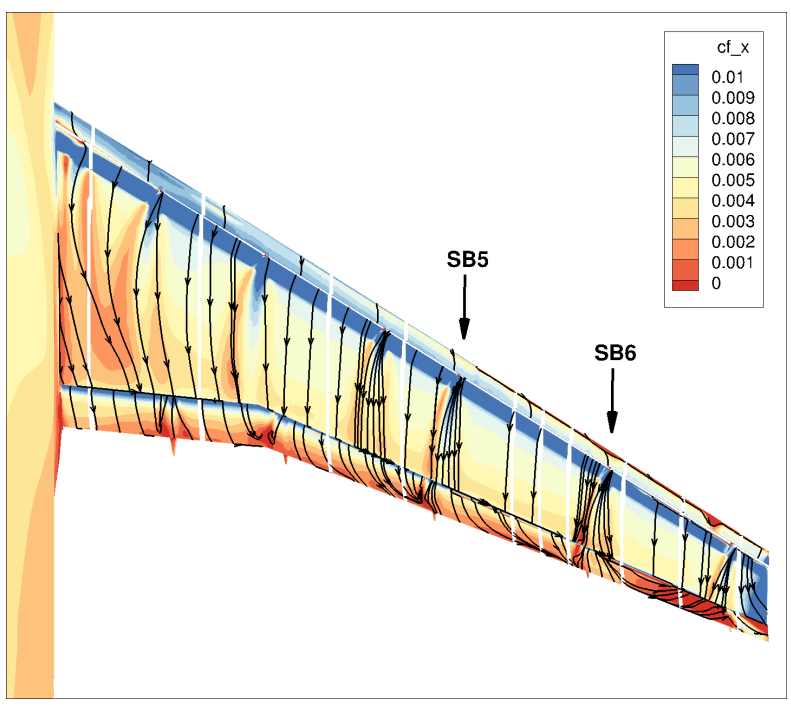

(c) Grid D, $\alpha=18.5^{\circ}$

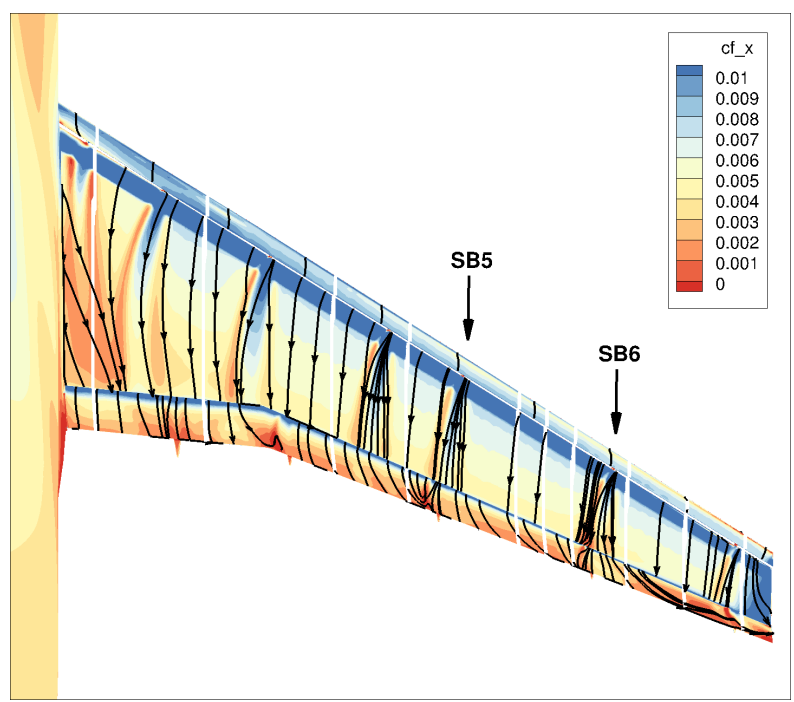

(e) Grid C, $\alpha=18.5^{\circ}$

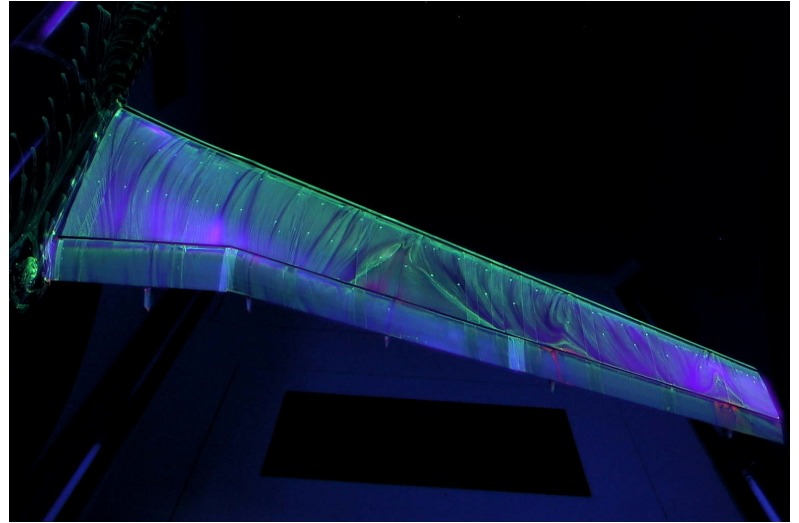

(b) Exp., $\alpha=21.0^{\circ}$

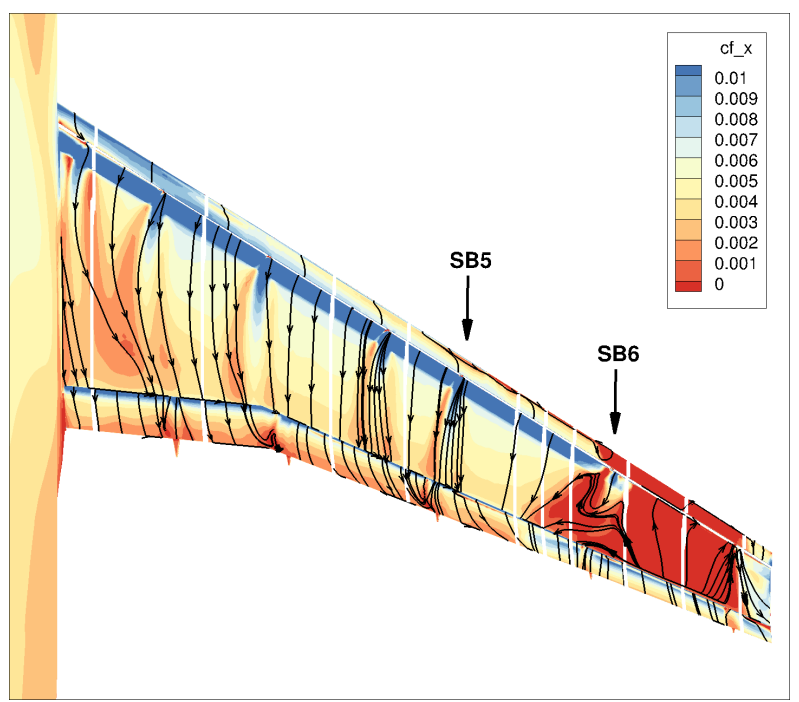

(d) Grid D, $\alpha=21.0^{\circ}$

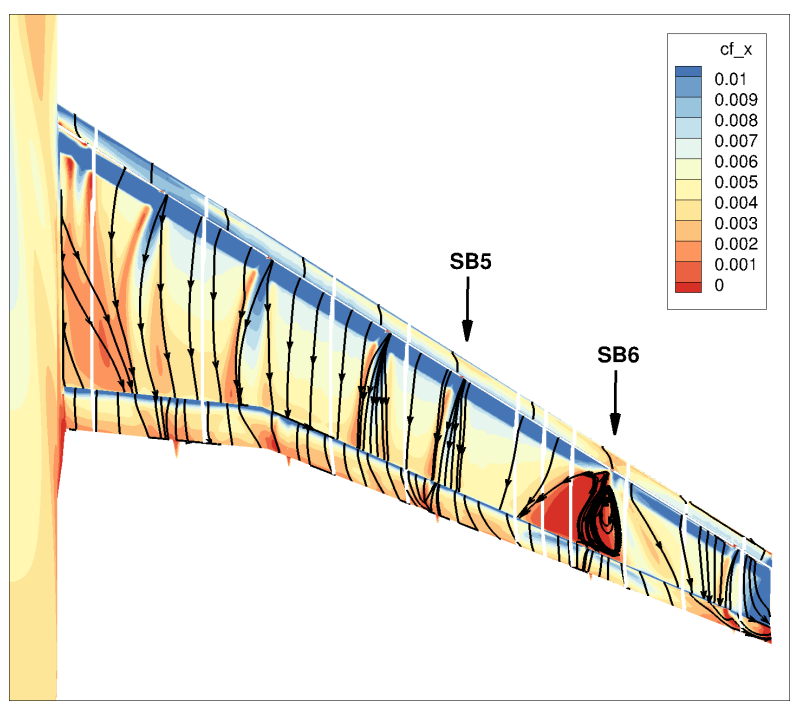

(f) Grid C, $\alpha=21.0^{\circ}$

Figure 12. Comparison of low $R e_{C}$ Config 4 with SA-RC-QCR2000 surface restricted streamlines and $C_{f}$ near stall (white lines pressure measurement stations). 

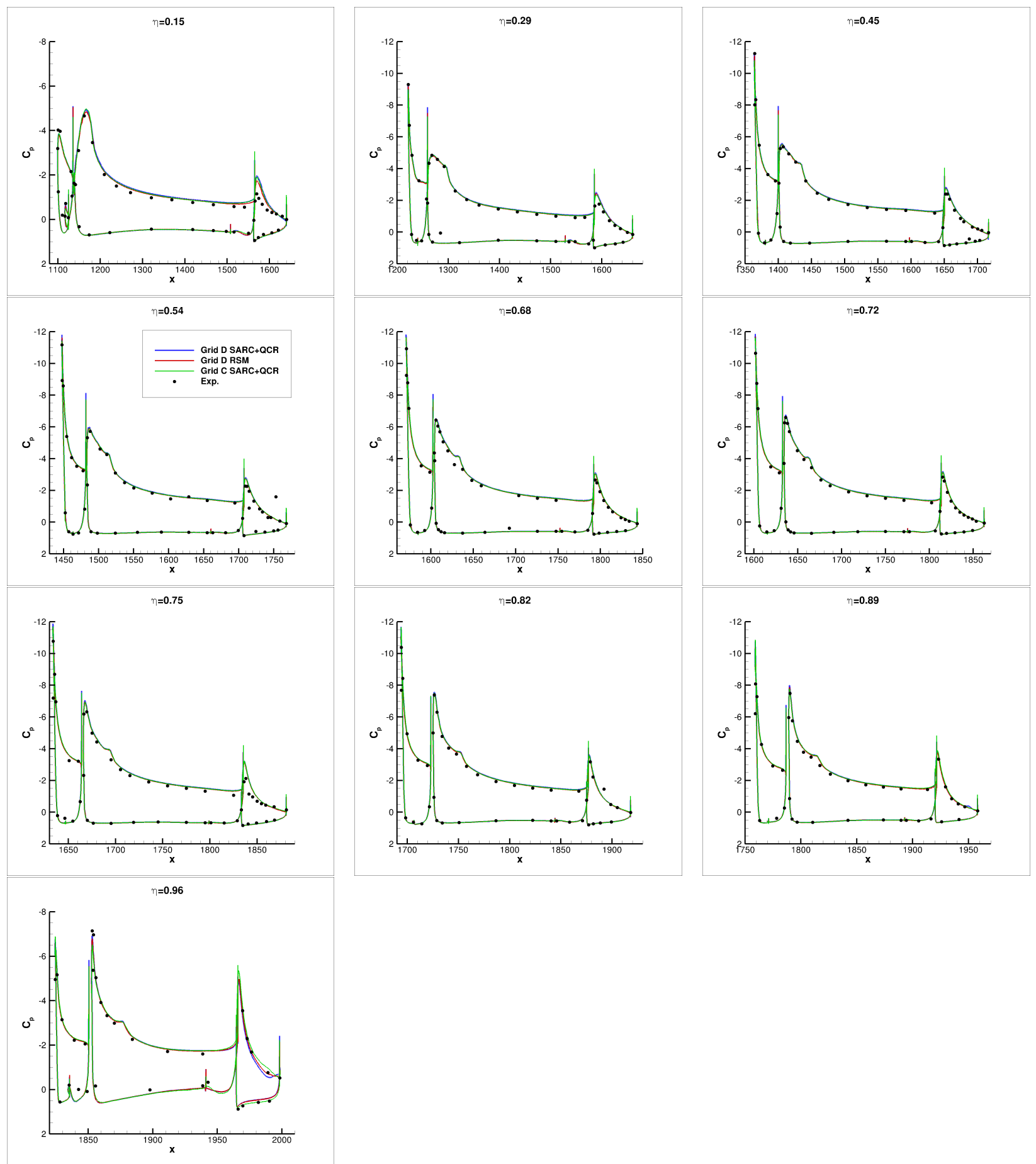

Figure 13. Comparison of high $R e_{C}$ Config 4 surface pressures at $\alpha=18.5^{\circ}$. 

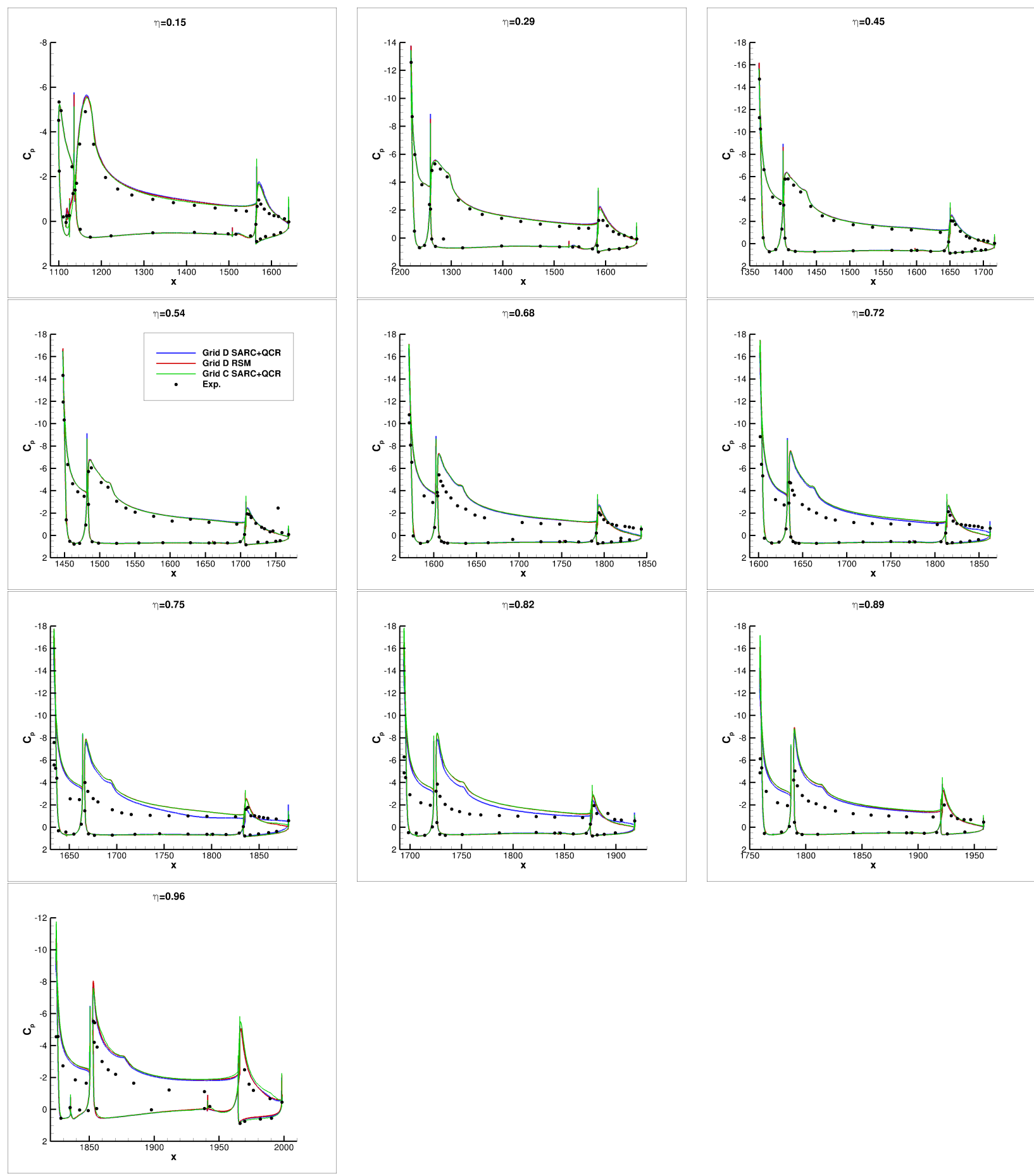

Figure 14. Comparison of high $\operatorname{Re}_{C}$ Config 4 surface pressures at $\alpha=22.0^{\circ}$. 


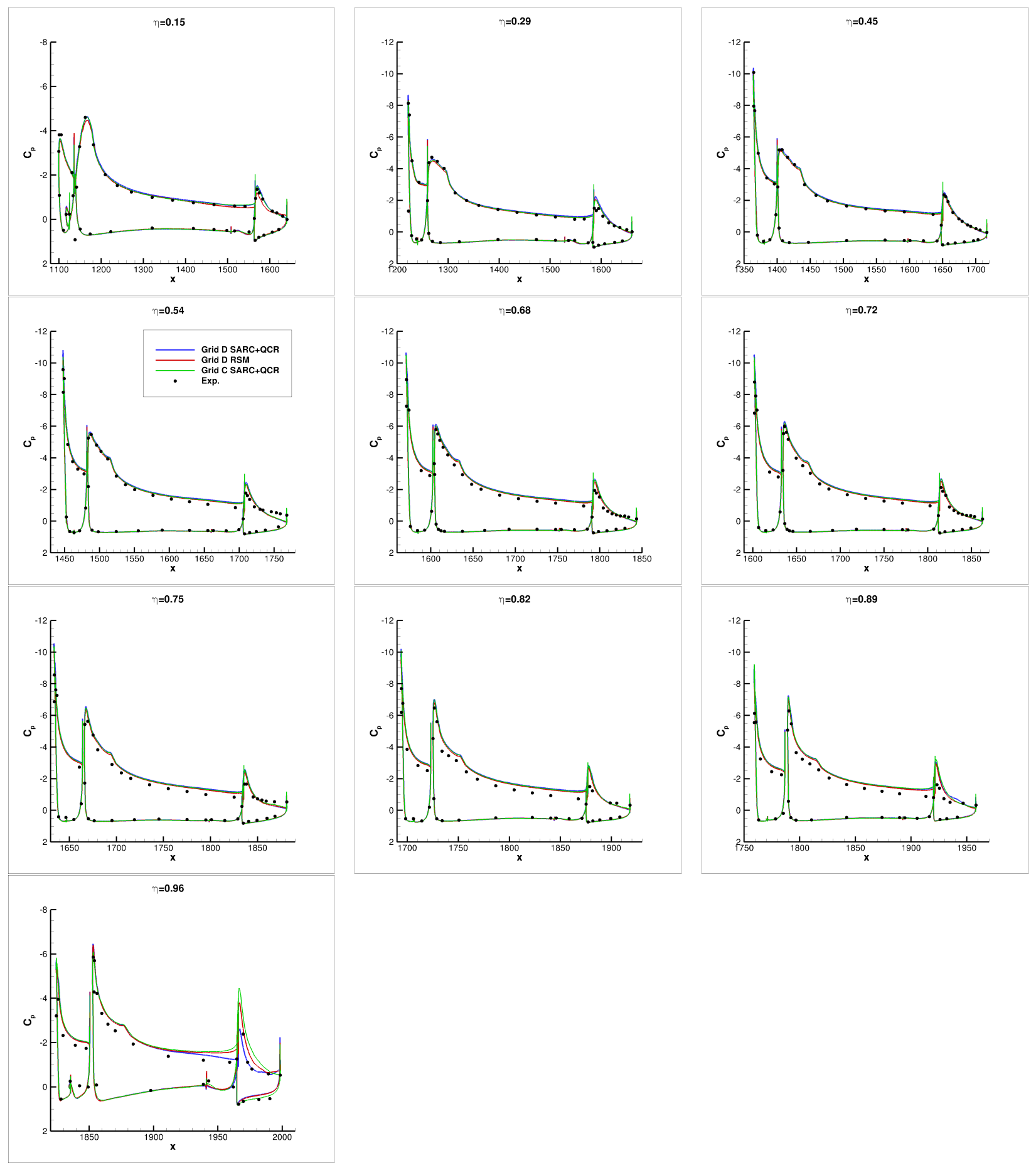

Figure 15. Comparison of low $R e_{C}$ Config 4 surface pressures at $\alpha=18.5^{\circ}$. 

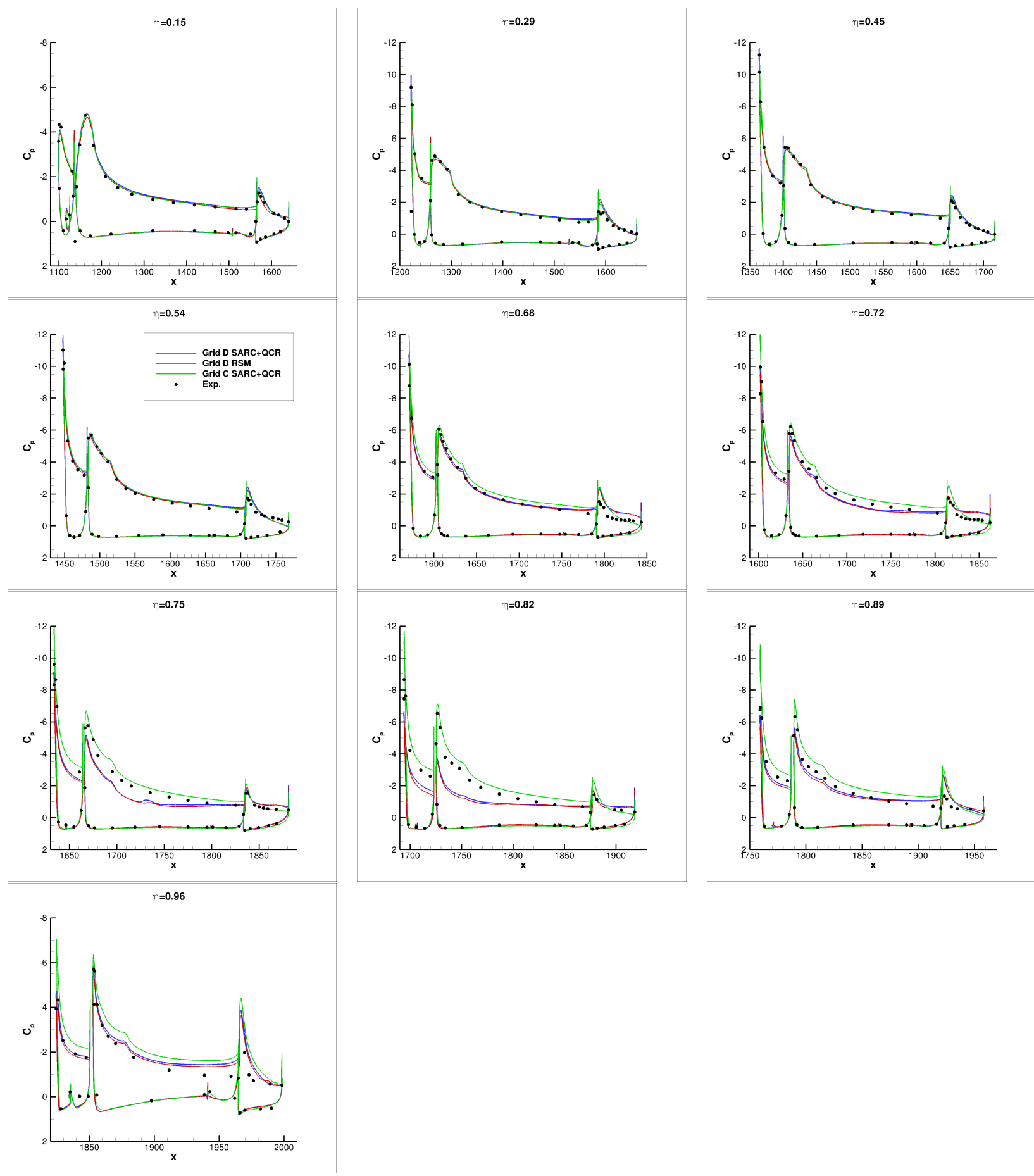

Figure 16. Comparison of low $\operatorname{Re}_{C}$ Config 4 surface pressures at $\alpha=20^{\circ}$. 


\section{References}

${ }^{1}$ Eisfeld, B. and Brodersen, O., "Advanced Turbulence Modelling and Stress Analysis for the DLR-F6 Configuration," AIAA Paper 2005-4727, 2005.

${ }^{2}$ Eisfeld, B., Rumsey, C., and Togiti, V., "Second-Moment RANS Model Verification and Validation using the Turbulence Modeling Resource Website (Invited)," AIAA Paper 2015-2924, June 2015.

${ }^{3}$ Menter, F. R., "Two-Equation Eddy-Viscosity Turbulence Models for Engineering Applications," AIAA Journal, Vol. 32, No. 8, Aug. 1994, pp. 1598-1605.

${ }^{4}$ Rumsey, C. L., "Application of Reynolds Stress Models to Separated Aerodynamic Flows," Differential Reynolds Stress Modeling for Separating Flows in Industrial Aerodynamics, B. Eisfeld editor, Springer, 2015, pp. 19-37.

${ }^{5}$ Wilcox, D. C., Turbulence Modeling for CFD, DCW Industries, Inc., 2006.

${ }^{6}$ Launder, B. E., Reece, G. J., and Rodi, W., "Progress in the Development of a Reynolds-Stress Turbulence Closure Verification and Validation of a Second-Moment-Closure Model," Journal of Fluid Mechanics, Vol. 68, No. 3, 1975, pp. 537566.

${ }^{7}$ Rodio, J. J., Xiao, X., Hassan, H. A., and Rumsey, C. L., "NASA Trapezoidal-Wing Simulation Using Stress- $\omega$, and Oneand Two-Equation Turbulence Models," AIAA Journal of Aircraft, Vol. 52, No. 4, 2015, pp. 1189-1200.

${ }^{8}$ Thompson, K. B. and Hassan, H. A., "Simulation of a Variety of Wings using a Reynolds Stress Model," AIAA Paper 2014-2192, 2014.

${ }^{9}$ Spalart, P. R. and Allmaras, S. R., "A One-Equation Turbulence Model for Aerodynamic Flows," Recherche Aerospatiale, Vol. 1, No. 1, 1994, pp. 5-21.

${ }^{10}$ Rumsey, C. L. and Slotnick, J. P., "Overview and Summary of the Second AIAA High-Lift Prediction Workshop," AIAA Journal of Aircraft, Vol. 52, No. 4, 2015, pp. 1006-1025.

${ }^{11}$ Lee-Rausch, E. M., Rumsey, C. L., and Park, M. A., "Grid-Adapted FUN3D Computations for the Second High-Lift Prediction Workshop," AIAA Journal of Aircraft, Vol. 52, No. 4, July-August 2015, pp. 1098-1111.

${ }^{12}$ Eisfeld, B., Rumsey, C. L., and Togiti, V., "Verification and Validation of a Second-Moment-Closure Model," AIAA Journal, article in advance, doi: 10.2514/1.J054718, 2016.

${ }^{13}$ Speziale, C. G., Sarkar, S., and Gatski, T. B., "Modelling the Pressure-Strain Correlation of Turbulence: An Invariant Dynamical Systems Approach," Journal of Fluid Mechanics, Vol. 227, 1991, pp. 245-272. 2649.

${ }^{14}$ Daly, B. J. and Harlow, F. H., "Transport Equations in Turbulence," Physics of Fluids, Vol. 13, No. 11, 1970, pp. 2634-

${ }^{15}$ Shir, C. C., "A Preliminary Numerical Study of Atmospheric Turbulent Flows in the Idealized Planetary Boundary Layer," Journal of Atmospheric Science, Vol. 30, No. 10, 1972, pp. 1327-1339.

${ }^{16}$ Togiti, V. and Eisfeld, B., "Assessment of g-Equation Formulation for a Second-Moment Reynolds Stress Turbulence Model," AIAA Paper 2015-2925, June 2015.

${ }^{17}$ Shur, M. L., Strelets, M. K., Travin, A. K., and Spalart, P. R., "Turbulence Modeling in Rotating and Curved Channels: Assessing the Spalart-Shur Correction," AIAA Journal, Vol. 38, No. 5, 2000, pp. 784-792.

${ }^{18}$ Spalart, P. R., "Strategies for Turbulence Modelling and Simulation," International Journal of Heat and Fluid Flow, Vol. 21, 2000, pp. 252-263.

${ }^{19}$ Krist, S. L., Biedron, R. T., and Rumsey, C. L., "CFL3D User's Manual (Version 5.0)," NASA TM 208444, NASA Langley Research Center, June 1998.

${ }^{20}$ Roe, P. L., "Approximate Riemann Solvers, Parameter Vectors, and Difference Schemes," Journal of Computational Physics, Vol. 43, 1981, pp. 357-372.

${ }^{21}$ Anderson, W. K. and Bonhaus, D. L., "An Implicit Upwind Algorithm for Computing Turbulent Flows on Unstructured Grids," Computers and Fluids, Vol. 23, No. 1, 1994, pp. 1-22.

${ }^{22}$ Anderson, W. K., Rausch, R. D., and Bonhaus, D. L., "Implicit/Multigrid Algorithm for Incompressible Turbulent Flows on Unstructured Grids," 12th Computational Fluid Dynamics Conference, San Diego, CA, AIAA Paper 95-1740-CP, June 1995.

${ }^{23}$ Nielsen, E. J., Aerodynamic Design Sensitivities on an Unstructured Mesh Using the Navier-Stokes Equations and a Discrete Adjoint Formulation, Ph.D. thesis, Virginia Polytechnic Institute and State University, Dec. 1998.

${ }^{24}$ Venkatakrishnan, V., "Convergence to Steady State Solutions of the Euler Equations on Unstructured Grids with Limiters," Journal of Computational Physics, Vol. 118, No. 1, 1995, pp. 120-130.

${ }^{25}$ Diskin, B., Thomas, J. L., Nielsen, E. J., Nishikawa, H., and White, J. A., "Comparison of Node-Centered and CellCentered Unstructured Finite-Volume Discretizations: Viscous Fluxes," AIA A Journal, Vol. 48, No. 7, July 2010, pp. 1326-1338.

${ }^{26}$ Nielsen, E. J., Lu, J., Park, M. A., and Darmofal, D. L., "An Implicit, Exact Dual Adjoint Solution Method for Turbulent Flows on Unstructured Grids," Computers and Fluids, Vol. 33, No. 9, 2004, pp. 1131-1155.

${ }^{27}$ Schwamborn, D., Gardner, A., von Geyr, H., Krumbein, A., Ludeke, H., and Sturmer, A., "Development of the TAU-Code for Aerospace Applications," 50th NAL International Conference on Aerospace Science and Technology 2008-06-26 - 2008-06-28, 2008.

${ }^{28}$ Schmitt, V. and Charpin, F., "Pressure Distributions on the ONERA-M6-Wing at Transonic Mach Numbers," Experimental Data Base for Computer Program Assessment. Report of the Fluid Dynamics Panel Working Group 04, AGARD AR-138, May 1979.

${ }^{29}$ Mani, M., Ladd, J., Cain, A., and Bush, R., "An Assessment of One-and Two-Equation Turbulence Models for Internal and External Flows," AIAA Paper 1997-2010, June 1997.

${ }^{30}$ Anderson, W. K., Newman, J. C., Whitfield, D. L., and Nielsen, E. J., "Sensitivity Analysis for the Navier-Stokes Equations on Unstructured Meshes Using Complex Variables," AIAA Paper 99-3294, 1999.

${ }^{31}$ Pirzadeh, S. Z., "Three-Dimensional Unstructured Viscous Grids by the Advancing-Layers Method," AIAA Journal, Vol. 34, No. 1, Jan. 1996, pp. 43-49. 
${ }^{32}$ Cécora, R.-D., Radespiel, R., Eisfeld, B., and Probst, A., "Differential Reynolds-Stress Modeling for Aeronautics," AIAA Journal, Vol. 53, No. 3, March 2015, pp. 739-755.

${ }^{33}$ Rudnik, R., Huber, K., and Melber-Wilkending, S., "EUROLIFT Test Case Description for the 2nd High Lift Prediction Workshop," AIAA Paper 2012-2924, June 2012.

${ }^{34}$ Mavriplis, D., Long, M., Lake, T., and Langlois, M., "NSU3D Results for the Second AIAA High Lift Prediction Workshop," AIAA Paper 2014-0748, Jan. 2014.

${ }^{35}$ Rumsey, C. L., Slotnick, J. P., Long, M., Stuever, R. A., and Wayman, T. R., "Summary of the First AIAA CFD High Lift Prediction Workshop," AIAA Journal of Aircraft, Vol. 48, No. 6, Nov.-Dec. 2011, pp. 2068-2079.

${ }^{36}$ Park, M. A., Lee-Rausch, E. M., and Rumsey, C. L., "FUN3D and CFL3D Computations for the First High Lift Prediction Workshop," AIAA Paper 2011-0936, Jan. 2011. 